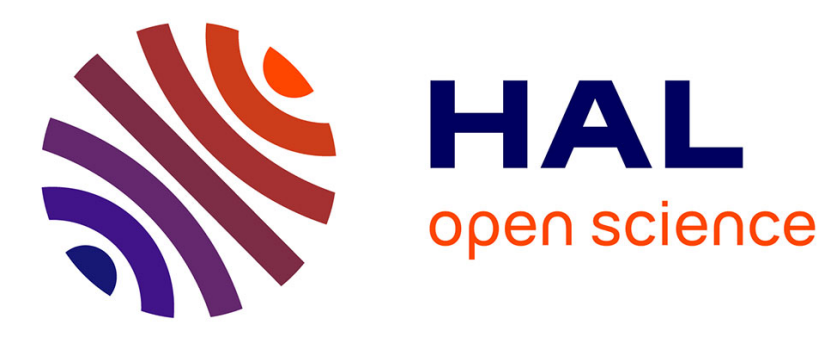

\title{
Gold-catalyzed synthesis of enantioenriched furfurylamines from amino acids
}

Benjamin Guieu, Myriam Le Roch, Michèle David, Nicolas Gouault

\section{To cite this version:}

Benjamin Guieu, Myriam Le Roch, Michèle David, Nicolas Gouault. Gold-catalyzed synthesis of enantioenriched furfurylamines from amino acids. Tetrahedron: Asymmetry, 2015, 26 (15-16), pp.868875. 10.1016/j.tetasy.2015.06.014 . hal-01188233

\section{HAL Id: hal-01188233 \\ https://hal-univ-rennes1.archives-ouvertes.fr/hal-01188233}

Submitted on 4 Nov 2015

HAL is a multi-disciplinary open access archive for the deposit and dissemination of scientific research documents, whether they are published or not. The documents may come from teaching and research institutions in France or abroad, or from public or private research centers.
L'archive ouverte pluridisciplinaire HAL, est destinée au dépôt et à la diffusion de documents scientifiques de niveau recherche, publiés ou non, émanant des établissements d'enseignement et de recherche français ou étrangers, des laboratoires publics ou privés. 


\title{
Gold-catalyzed synthesis of enantioenriched furfurylamines from amino
} acids.

\author{
Benjamin Guieu, Myriam Le Roch, Michèle David and Nicolas Gouault* \\ Equipe PNSCM, UMR 6226, Université de Rennes 1, 2 avenue du Pr. Léon Bernard 35043 Rennes Cedex, \\ France. \\ E-mail : nicolas.gouault@univ-rennes1.fr
}

\section{Abstract}

A convenient gold-catalyzed asymmetric synthesis of polysubstituted furfurylamines starting from amino acids has been achieved. The cyclization proceeded under mild conditions and generally provided the furan or iodofuran derivatives in good to excellent yields and high enantiomeric excess. Iodofurans were validated as good intermediates for classical organometallic coupling reactions.

\section{Introduction}

Polysubstituted furans are key structural units found in many natural products ${ }^{1}$ and pharmaceuticals. ${ }^{2}$ Thus, several methods for their synthesis have been developed in the past few years, ${ }^{3}$ including transition-metal-mediated cyclizations and cycloisomerizations. ${ }^{4}$

Optically active 2-aminomethyl-furans have important applications as chiral ligands or organocatalysts, ${ }^{5}$ and as building blocks for various biologically active molecules ${ }^{6}$ and provide access to many nitrogen containing compounds such as piperidines or aza-sugars -by the aza-Achmanowicz rearrangement- ${ }^{7}$ or amino acids by oxidative cleavage. ${ }^{8}$ However, few stereoselective syntheses of chiral furfurylamines have been described and most of them involve the modification of substituted furans, such as the asymmetric aminohydroxylation of vinylfuran or the enantioselective reduction of furanyl ketones. ${ }^{9}$ Thus, the search for efficient, convenient and highly stereoselective alternative synthetic routes is of great interest.

We have previously reported diverse gold-catalyzed approaches to nitrogen containing 5or 6- membered heterocycles from amino acids. ${ }^{10}$ We herein report an efficient synthetic route to some chiral di- and tri-substituted $\alpha$-aminomethylfuran derivatives from $\alpha$-aminoalkynyl1,2-diols prepared from commercially available amino-acids. Our synthetic approach is outlined in scheme 1 . 


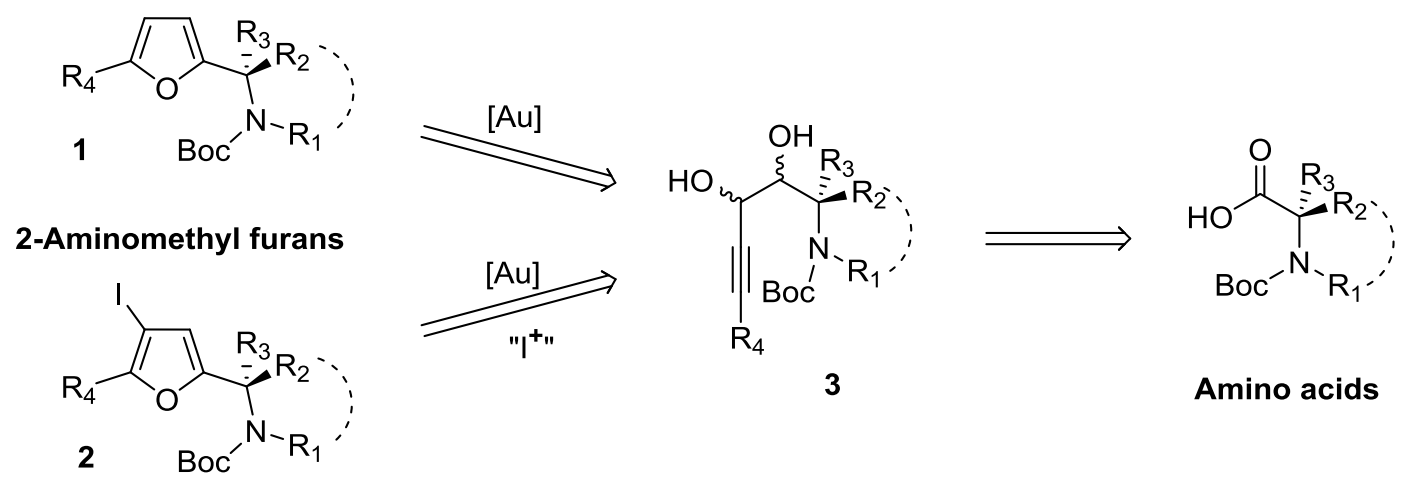

Scheme 1. Retrosynthetic strategy.

The structural diversity of commercially available enantiopure amino acids and the opportunity for late-stage diversification at intermediate diol $\mathbf{3}$ should allow rapid generation of enantioenriched 2-amino furan libraries by this strategy. One major advantage of gold catalyzed cyclization is minimal racemization under the very mild conditions.

\section{Results and discussion}

Our initial efforts focused on the synthesis of the key intermediates acetylenic diols $\mathbf{3}$, precursors of chiral 2-aminomethylfurans 1 or $\mathbf{2}$ by gold-catalyzed dehydrative cyclization. We first prepared aldehydes 4a-d on a gram scale in two steps from commercially available $N$-Boc protected amino acids. Amino acids were converted to their corresponding Weinreb amides using TBTU, DIEA and N,O-dimethylhydroxylamine hydrochloride in DMF, then reduced without any racemization using lithium aluminium hydride in diethylether at $-15^{\circ} \mathrm{C}$ according to a published procedure. ${ }^{11}$ The observed optical rotation of these derivatives 4 were in agreement with the literature. ${ }^{12}$

Intermediates enynes $\mathbf{6}$ were obtained from aldehydes $\mathbf{4}$ by one step (Wittig reaction, Method A) or two steps (Takai iodovinylation ${ }^{13}$ then Sonogashira coupling reaction, Method B) routes (Scheme 2).

Wittig reaction of $\mathbf{4 a - b}$ with 2-pentynyltriphenylphosphonium bromide and $n$-BuLi in THF afforded amino enynes 6a-b in moderate yield as a mixture of diastereoisomers (E/Z: 80/20). ${ }^{14}$ Method B is a more general strategy due to the wide variety of commercially available terminal alkynes. Trans vinyl iodides 5 were obtained by treatment of aldehydes $\mathbf{4}$ with iodoform in the presence of $\mathrm{CrCl}_{2},{ }^{15}$ and Sonogashira coupling ${ }^{16}$ of 1-pentyne or phenylacetylene in the presence of $\mathrm{PdCl}_{2}\left(\mathrm{PPh}_{3}\right)_{2}$ yielded enynes 6c-d (51-67 \% yield over two 
steps). The trans geometry of the vinyl iodides $\mathbf{5}$ was confirmed by the coupling constant of the two vinylic protons $(J=14.5 \mathrm{~Hz})$.

Method A (Wittig Reaction)
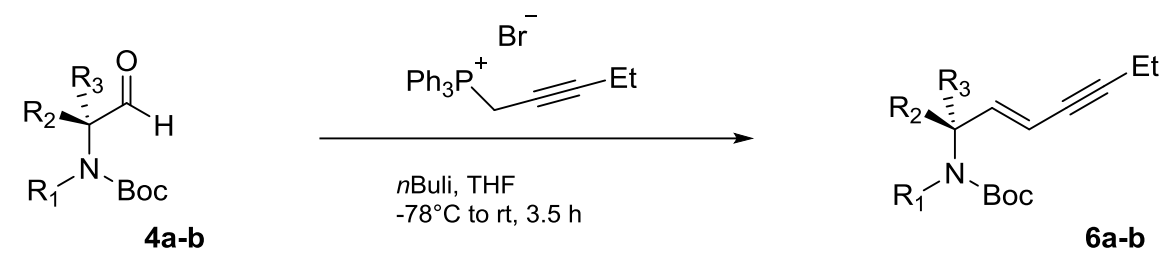

4a: $\mathrm{R}_{1}, \mathrm{R}_{3}=\mathrm{H}, \mathrm{R}_{2}=\mathrm{CH}_{2} \mathrm{OBn}$

6a: $56 \%, E / Z=80 / 20$

4b: $\mathrm{R}_{1}, \mathrm{R}_{2}=\mathrm{H}, \mathrm{R}_{3}=\mathrm{CH}_{2} \mathrm{OBn}$

6b: $56 \%, E / Z=80 / 20$

Method B (lodovinylation and Sonogashira coupling reaction)

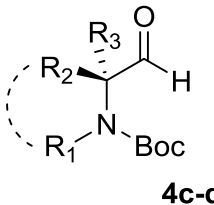

4c: $R_{1}, R_{2}=H, R_{3}=B n$

4d: $\mathrm{R}_{1}, \mathrm{R}_{2}=-\left(\mathrm{CH}_{2}\right)_{3^{-}}, \mathrm{R}_{3}=\mathrm{H}$

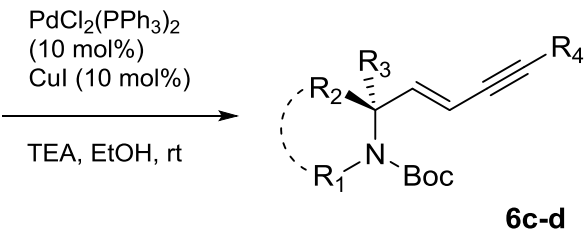

6c: $\mathrm{R}_{4}=n \operatorname{Pr}, 51 \%, E / Z=90 / 10$ 6d: $R_{4}=P h, 67 \%, E / Z=90 / 10$

Scheme 2. Synthesis of enyne intermediates 6.

We next focused on the dihydroxylation ${ }^{17}$ of alkenes $\mathbf{6 a - d}$ to obtain dihydroxy alkynes $\mathbf{3}$ (Scheme 3). Osmium tetroxide and $\mathrm{N}$-methylmorpholine $\mathrm{N}$-oxide failed to deliver the expected diols 3, but modified Sharpless asymmetric dihydroxylation conditions with additional methane sulfonamide ${ }^{18}$ allowed the isolation of the diols 3a-d in a range of 72-79 $\%$ yield. The use of AD-mix- $\beta$-easier to manipulate- and methane sulfonamide in $t$ $\mathrm{BuOH}: \mathrm{H}_{2} \mathrm{O}(1: 1)$ gave best yields.<smiles>[R]C#CC=CC1([R])[R]([R])CCCN1[R17]</smiles>

6a-d
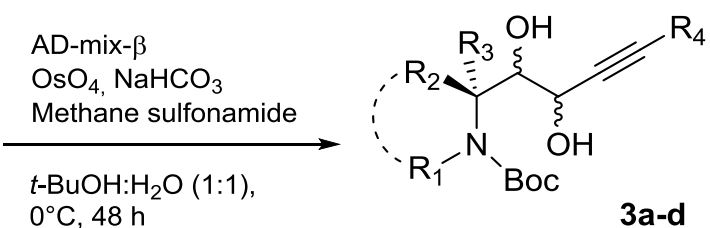

3a: $76 \%$

3b: $72 \%$

3c: $75 \%$

3d: $79 \%$

Scheme 3. Dihydroxylation of intermediates 6. 
With these diols 3 in hands, we next turned our attention to their gold-catalyzed dehydrative cyclization into di-substituted furans $\mathbf{1}$ and 4-iodofurans $\mathbf{2}$ using reaction conditions that were successful in our previous work (Scheme 4). ${ }^{10}$

In order to validate this cyclization, diol 3a was treated with $\mathrm{PPh}_{3} \mathrm{AuCl}(1 \mathrm{~mol} \%)$ and $\operatorname{AgSbF}_{6}(1 \mathrm{~mol} \%)$ in 1,2-dichloroethane (1,2-DCE) at room temperature to afford furan $1 \mathrm{a}$ in $89 \%$ yield in $1 \mathrm{~h}$. As expected, no reaction was observed when $\mathrm{AgSbF}_{6}$ or $\mathrm{PPh}_{3} \mathrm{AuCl}$ catalysts were used separately.

Iodocyclization was next investigated. The reaction of 3a with NIS or $\mathrm{I}_{2}$ as electrophilic halogen sources in the absence of a catalyst returned either starting 3a or complex mixtures with only traces 2a. Catalytic iodocyclization under conditions previously developed in our laboratory ${ }^{19}$ revealed that the use of $\mathrm{PPh}_{3} \mathrm{AuCl}(1 \mathrm{~mol} \%)$ in the presence of $\mathrm{AgSbF}_{6}(1 \mathrm{~mol} \%)$, with NIS (1.2 eq) as electrophile, in 1,2-DCE at room temperature, afforded furan 2a in $62 \%$ yield in $1 \mathrm{~h}$ (Scheme 3). Similar treatment of enantiomeric $\mathbf{3 b}$ allowed purity measurement of 1a-b and 2a-b (93-96\% ee) by chiral HPLC, which indicated minimal ee erosion during the sequence.

Various $\alpha, \beta$-dihydroxyalkynes $\mathbf{3}$ were submitted to our reaction conditions to generate series of disubstituted furans $\mathbf{1}$ and iodofurans $\mathbf{2}$ in moderate to excellent yields (Scheme 4).
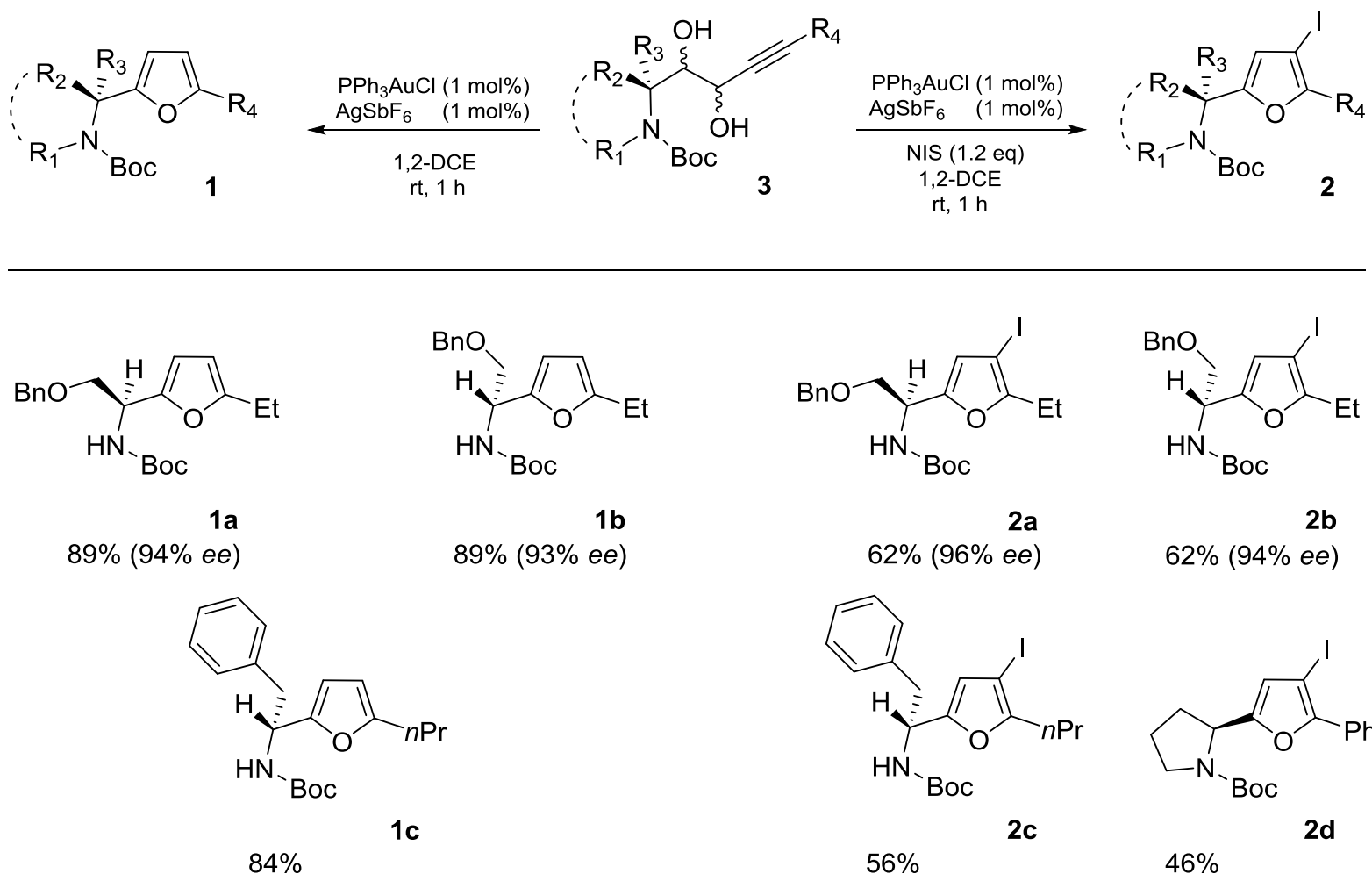

$89 \%(93 \%$ ee $)$
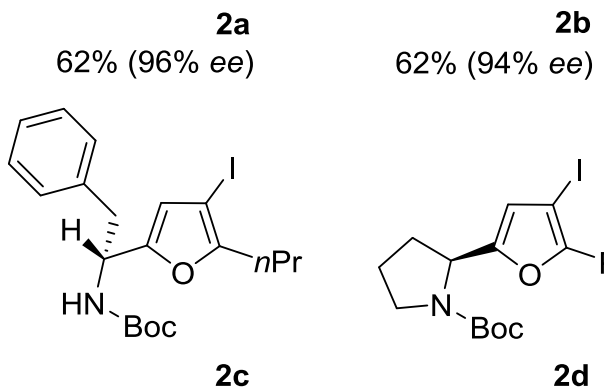

$56 \%$

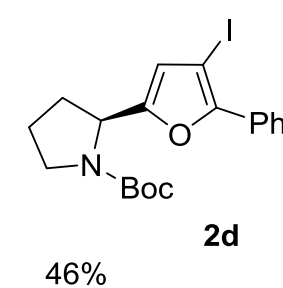

Scheme 4. Gold-catalyzed cyclization of substrates $\mathbf{3}$ to furans $\mathbf{1}$ and 2. 
The value of iodo-furans $\mathbf{2}$ as chiral building blocks was demonstrated by further manipulation using Suzuki-Miyaura, ${ }^{20}$ Heck, $^{21}$ Sonogashira $^{22}$ and Buchwald-Hartwig ${ }^{23}$ couplings (Scheme 5). Compounds 7-9 were obtained in excellent isolated yields (87-94\%) by Suzuki-Miyaura cross-coupling reaction of $\mathbf{2}$ with arylboronic acids. Heck and Sonogashira reactions of $\mathbf{2 c}$ with ethyl acrylate or phenylacetylene respectively provided adducts $\mathbf{1 0}$ and $\mathbf{1 1}$ in $60 \%$ and $94 \%$ isolated yields. Finally, Buchwald-Hartwig amination of 2d with tertbutylcarbamate gave the corresponding aminofuran 12, albeit in low yield.

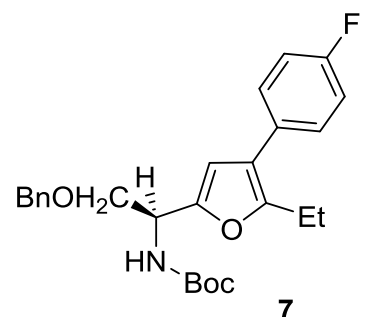

$87 \%(96 \%$ ee $)$

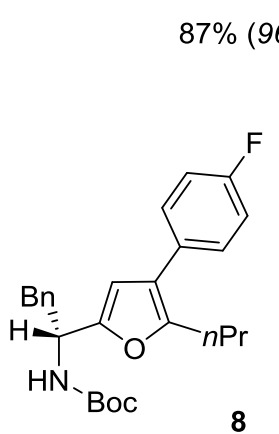

$95 \%(99 \%$ ee $)$

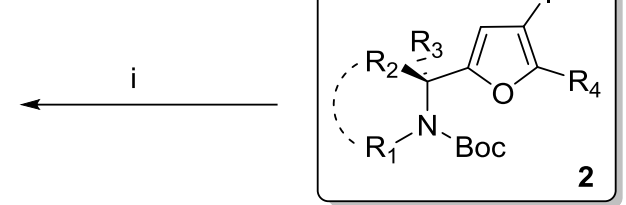

2

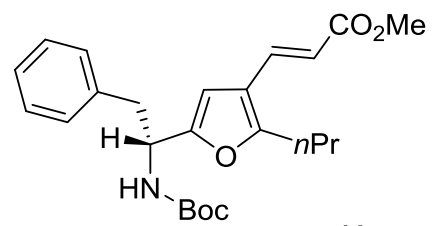

10

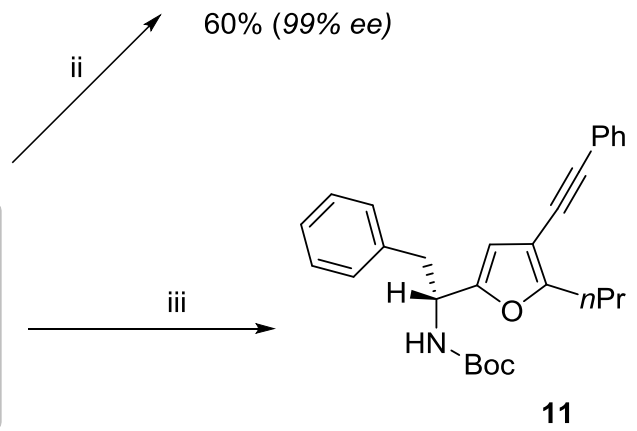

$94 \%$ (99\% ee)
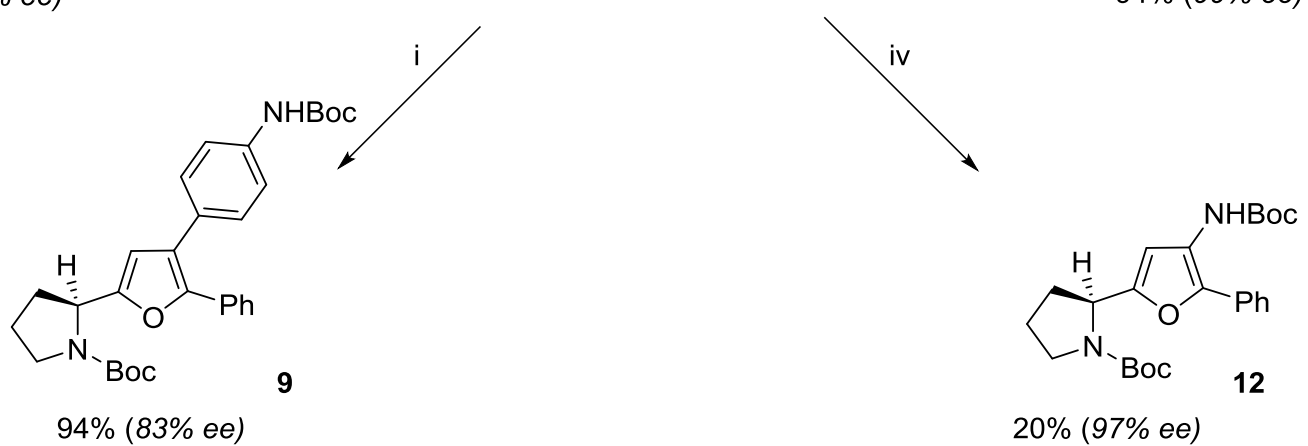

Scheme 5. Reagents and conditions: Suzuki-Miyaura coupling reaction (i) $\mathrm{Ar}-\mathrm{B}(\mathrm{OH})_{2}, \mathrm{~K}_{3} \mathrm{PO}_{4}$, S-Phos $(10 \mathrm{~mol} \%), \mathrm{Pd}(\mathrm{OAc})_{2}(5 \mathrm{~mol} \%)$, toluene, $80^{\circ} \mathrm{C}, 3 \mathrm{~h}$; Heck coupling reaction (ii) Methyl acrylate, TEA, $\mathrm{Pd}\left(\mathrm{PPh}_{3}\right)_{4}(10 \mathrm{~mol} \%)$, DMF, $80^{\circ} \mathrm{C}, 2 \mathrm{~h}$; Sonogashira coupling reaction (iii) Phenylacetylene, $\mathrm{PdCl}_{2}\left(\mathrm{PPh}_{3}\right)_{2}(1 \mathrm{~mol} \%), \mathrm{CuI}(2 \mathrm{~mol} \%), \mathrm{TEA}, \mathrm{rt}, 4 \mathrm{~h}$; Buchwald-Hartwig coupling reaction (iv) tertButyl carbamate, $\mathrm{Cs}_{2} \mathrm{CO}_{3}, \mathrm{CuI}(20 \mathrm{~mol} \%), \mathrm{N}^{-N^{\prime}-D M E D A}(40 \mathrm{~mol} \%)$, toluene, $90^{\circ} \mathrm{C}, 18 \mathrm{~h}$.

Access to fully deprotected $\beta$-amino alcohol derivatives by our strategy was then investigated. Efficient hydrogenolysis of the benzyl groups in 1a and $\mathbf{7}$ was accomplished 
with cyclohexene in the presence of Pearlman's catalyst to give $\mathbf{1 3}$ and $\mathbf{1 4}$ with an excellent enantiomeric excess (Scheme 6).

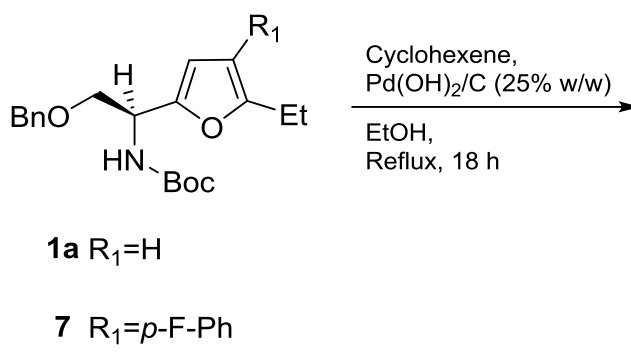

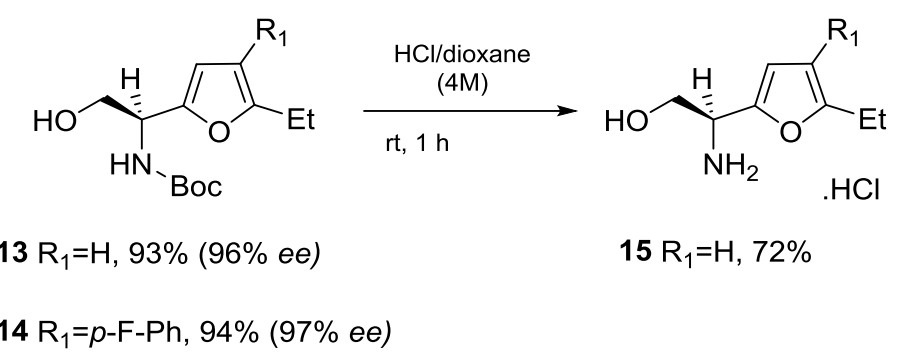

Scheme 6. Removal of protecting groups and access to amino-alcohol derivatives

Finally, $N$-Boc deprotection of $\mathbf{1 3}$ with $4 \mathrm{M} \mathrm{HCl}$ in dioxane proceeded smoothly to give $\beta$ amino alcohol 15 in $72 \%$ yield.

\section{Conclusion}

In conclusion, we have reported a convenient gold-catalyzed approach for the synthesis of polysubstituted aminomethyl-furan derivatives from chiral acetylenic diol intermediates. The reaction proceeds under mild conditions, generally providing furan products in good to excellent yields and enantiopurity. Such compounds may have applications as chiral ligands, organocatalysts and pharmaceuticals.

\section{Experimental}

\subsection{General}

All reagents of high quality were purchased from commercial suppliers, and used without further purification. All reactions requiring anhydrous conditions were performed under an argon atmosphere using oven dried glassware. DMF and THF were distilled from $\mathrm{CaH}_{2}$ and $\mathrm{Na}$ /benzophenone, respectively. ${ }^{1} \mathrm{H}$ and ${ }^{13} \mathrm{C} \mathrm{NMR}$ were recorded at 300 and $75 \mathrm{MHz}$ respectively on a Bruker AM 300 spectrometer, using $\mathrm{CDCl}_{3}$ (and TMS as internal standard). $\delta$ values are given in parts per million (ppm), coupling constants ( $J$ values) are given in Hertz 
$(\mathrm{Hz})$, and multiplicity of signals are reported as follows: s, singlet; d, doublet; t, triplet; $\mathrm{q}$, quadruplet; m, multiplet; bs, broad singlet. Thin layer chromatography was performed using precoated silica gel plate $(0.2 \mathrm{~mm}$ thickness $)$. Infrared spectra were recorded using an Universal Attenuated Total Reflectance Accessory (UATR). All melting points are uncorrected. Optical rotations were measured at the sodium D line on a digital polarimeter at ambient temperature.

\subsection{Typical procedure for the preparation of vinyl iodides 5}

Chromium(II) chloride (2.22 g, $18.0 \mathrm{mmol})$ was suspended in dry THF (25 mL) at room temperature under argon. A solution of aldehyde $4 \mathbf{c}$ or $\mathbf{4 d}(4.0 \mathrm{mmol})$ and iodoform $(2.37 \mathrm{~g}$, $6.0 \mathrm{mmol})$ in THF $(25 \mathrm{~mL})$ was added dropwise. After stirring in the dark at room temperature for $2 \mathrm{~h}$, the reaction mixture was hydrolysed with a satured solution of $\mathrm{NH}_{4} \mathrm{Cl}(50$ $\mathrm{mL})$ and extracted with diethylether $(3 \times 40 \mathrm{~mL})$. The combined organic layers were washed with brine, then dried over magnesium sulfate, concentrated and purified by silica gel chromatography (2\% ethyl acetate in dichloromethane) to afford the products $\mathbf{5 c}$ and $\mathbf{5 d}$ as a mixture of isomers $\mathrm{E} / \mathrm{Z}$, ratio: 90/10.

\subsection{1 tert-Butyl (R,E)-(4-iodo-1-phenylbut-3-en-2-yl)carbamate 5c}

$970 \mathrm{mg}(65 \%)$. Data of the separated major isomer E: white solid, $\mathrm{mp}=108-109{ }^{\circ} \mathrm{C}$; $[\alpha]_{\mathrm{D}}^{22}=+23.8\left(c\right.$ 1.04, $\mathrm{CH}_{2} \mathrm{Cl}_{2}$ ); IR (UATR): 3351, 1687, $1612 \mathrm{~cm}^{-1} ;{ }^{1} \mathrm{H}$ NMR $(300 \mathrm{MHz}$, $\left.\mathrm{CDCl}_{3}\right): \delta 7.34-7.21(\mathrm{~m}, 3 \mathrm{H}), 7.17-7.14(\mathrm{~m}, 2 \mathrm{H}), 6.47(\mathrm{dd}, J=14.5 \mathrm{~Hz}, J=5.9 \mathrm{~Hz}, 1 \mathrm{H}), 6.19$ $(\mathrm{dd}, J=14.5 \mathrm{~Hz}, \quad J=1.2 \mathrm{~Hz}, 1 \mathrm{H}), 4.47$ (bs, 1H), 4.39 (bs, 1H), 2.82 (bd, $J=6.6 \mathrm{~Hz}, 2 \mathrm{H})$, $1.40(\mathrm{~s}, 9 \mathrm{H}) ;{ }^{13} \mathrm{C} \mathrm{NMR}\left(75 \mathrm{MHz}, \mathrm{CDCl}_{3}\right): \delta 155.0,145.5,136.7,129.6,128.7,126.9,80.0$, 77.5, 55.9, 41.2, 28.4; HRMS (ESI, m/z): Calcd. for $\mathrm{C}_{15} \mathrm{H}_{20} \mathrm{NO}_{2} \mathrm{INa}$ : 396.0437, found $[\mathrm{M}+\mathrm{Na}]^{+}: 396.0437$.

\subsection{2 (S,E/Z)-2-(2-Iodovinyl)pyrrolidine-1-carboxylic acid tert-Butyl ester 5d}

$870 \mathrm{mg}$ (67\%), pale yellow oil. Data of the mixture of isomers: IR (UATR): 1687, 1607

$\mathrm{cm}^{-1}$; spectroscopic data for the unseparated major isomer E: ${ }^{1} \mathrm{H} \mathrm{NMR}\left(300 \mathrm{MHz}, \mathrm{CDCl}_{3}, 50\right.$ $\left.{ }^{\circ} \mathrm{C}\right): \delta 6.42(\mathrm{dd}, J=14.4 \mathrm{~Hz}, J=6.5 \mathrm{~Hz}, 1 \mathrm{H}), 6.14(\mathrm{~d}, J=14.4 \mathrm{~Hz}, 1 \mathrm{H}), 4.24(\mathrm{bs}, 1 \mathrm{H}), 3.38$ $(\mathrm{t}, J=6.6 \mathrm{~Hz}, 2 \mathrm{H}), 2.05-1.90(\mathrm{~m}, 1 \mathrm{H}), 1.89-1.69(\mathrm{~m}, 3 \mathrm{H}), 1.44(\mathrm{~s}, 9 \mathrm{H}) ;{ }^{13} \mathrm{C}$ NMR $(75 \mathrm{MHz}$, 
$\left.\mathrm{CDCl}_{3}, 50{ }^{\circ} \mathrm{C}\right): \delta 154.5,146.5,79.7,76.1,61.3,46.4,31.4,28.7,23.3 ;$ HRMS (ESI, m/z):

Calcd. for $\mathrm{C}_{11} \mathrm{H}_{18} \mathrm{NO}_{2} \mathrm{INa}$ : 346.0280, found [M+Na] $]^{+}$: 346.0283 .

\subsection{Typical procedure for the preparation of enynes 6}

\subsubsection{From aldehydes $4($ Method $A)$}

To a stirred suspension of 2-pentynyltriphenylphosphonium bromide ${ }^{14}(1.43 \mathrm{~g}, 3.5 \mathrm{mmol})$ in $18 \mathrm{~mL}$ of dry $\mathrm{THF}$ at $-78{ }^{\circ} \mathrm{C}$ under argon atmosphere was added dropwise $1.7 \mathrm{~mL}$ (3.5 mmol) of a $2.1 \mathrm{M}$ solution of n-butyllithium in hexane. After stirring at $-78{ }^{\circ} \mathrm{C}$ for $1 \mathrm{~h}$, a solution of $N$-Boc-L-serinal 4a or $N$-Boc-D-serinal $4 \mathbf{b}(0.98 \mathrm{~g}, 3.5 \mathrm{mmol})$ in $15 \mathrm{~mL}$ of dry THF was added. After stirring at $-78{ }^{\circ} \mathrm{C}$ for $30 \mathrm{~min}$, the mixture was allowed to warm to room temperature over $2 \mathrm{~h}$. Then $40 \mathrm{~mL} \mathrm{H}_{2} \mathrm{O}$ was added and the mixture was extracted with diethylether $(3 \times 40 \mathrm{~mL})$. The combined organic extracts were washed with brine, dried over magnesium sulfate and concentrated. Chromatography on silica gel, eluting with $2 \%$ diethylether in dichloromethane gave $\mathbf{6 a}$ or $\mathbf{6 b}$.

\subsubsection{1 tert-Butyl $(S, E)-(1-(b e n z y l o x y) o c t-3-e n-5-y n-2-y l) c a r b a m a t e ~ 6 a$}

$650 \mathrm{mg}(56 \%)$, pale yellow oil, as a mixture of $E / Z$ isomers, ratio: 80/20. Data of the separated major $E$ isomer: $[\alpha]_{\mathrm{D}}^{22}=-29.0$ ( $c$ 1.05, $\mathrm{CHCl}_{3}$ ); IR (HATR): 3337, 2216, 1698, 1633 $\mathrm{cm}^{-1} ;{ }^{1} \mathrm{H}$ NMR $\left(300 \mathrm{MHz}, \mathrm{CDCl}_{3}\right): \delta$ 7.39-7.27 (m, 5H), $6.03(\mathrm{dd}, J=15.9 \mathrm{~Hz}, J=6.1 \mathrm{~Hz}$, $1 \mathrm{H}), 5.68(\mathrm{dm}, J=15.9 \mathrm{~Hz}, 1 \mathrm{H}), 4.88(\mathrm{bs}, 1 \mathrm{H}), 4.52(\mathrm{~s}, 2 \mathrm{H}), 4.34(\mathrm{bs}, 1 \mathrm{H}), 3.54$ (dd, $J=9.5$ $\mathrm{Hz}, J=4.4 \mathrm{~Hz}, 1 \mathrm{H}), 3.47(\mathrm{dd}, J=9.5 \mathrm{~Hz}, J=4.5 \mathrm{~Hz}, 1 \mathrm{H}), 2.30(\mathrm{qd}, J=7.5 \mathrm{~Hz}, J=2.1 \mathrm{~Hz}$, $1 \mathrm{H}), 1.44(\mathrm{~s}, 9 \mathrm{H}), 1.15$ (t, $J=7.5 \mathrm{~Hz}, 3 \mathrm{H}) ;{ }^{13} \mathrm{C} \mathrm{NMR}\left(75 \mathrm{MHz}, \mathrm{CDCl}_{3}\right): \delta 155.3,139.7,137.9$, 128.6, 127.9, 127.8, 111.8, 92.7, 79.8, 77.8, 73.4, 72.0, 52.1, 28.5, 14.0, 13.2; HRMS (ESI, $\mathrm{m} / \mathrm{z}$ ): Calcd. for $\mathrm{C}_{20} \mathrm{H}_{27} \mathrm{NO}_{3} \mathrm{Na}$ : 352.1888, found [M+Na] ${ }^{+}: 352.1884$.

\subsubsection{2 tert-Butyl (R,E)-(1-(benzyloxy)oct-3-en-5-yn-2-yl)carbamate $6 \mathrm{~b}$}

$650 \mathrm{mg}(56 \%)$, pale yellow oil. Data of the separated major $E$ isomer: $[\alpha]_{\mathrm{D}}^{22}=+27.1(c$ 0.93, $\mathrm{CHCl}_{3}$ ); spectral data and HRMS are identical with those reportated for $6 \mathbf{a}$. 


\subsubsection{From vinyl iodides 5 (Method B)}

To a degassed solution of vinyl iodide $\mathbf{5 c}$ or $\mathbf{5 d}(2.5 \mathrm{mmol})$ and triethylamine $(0.7 \mathrm{~mL}, 5$ $\mathrm{mmol})$ in $25 \mathrm{~mL}$ EtOH were added successively under argon atmoshere, $\mathrm{PdCl}_{2}\left(\mathrm{PPh}_{3}\right)_{2}(17.5$ $\mathrm{mg}, 0.025 \mathrm{mmol}, 1 \mathrm{~mol} \%), \mathrm{CuI}(23.8 \mathrm{mg}, 0.125 \mathrm{mmol}, 5 \mathrm{~mol} \%)$ and the appropriate alkyne ( $2.75 \mathrm{mmol}$ ). The mixture was stirred $72 \mathrm{~h}$ for $\mathbf{6 c}$ and $24 \mathrm{~h}$ for $\mathbf{6 d}$ at room temperature. After the reaction was completed $\left({ }^{1} \mathrm{H}\right.$ NMR analysis), $50 \mathrm{~mL} \mathrm{H}_{2} \mathrm{O}$ was added and the mixture was extracted with diethylether $(3 \times 40 \mathrm{~mL})$. The combined organic extracts were washed with brine, dried over magnesium sulfate and concentrated. Chromatography on silica gel, eluting with $20 \%$ cyclohexane in dichloromethane gave $\mathbf{6 c - d}$ as a mixture of $E / Z$ isomers, ratio:90/10.

\subsubsection{1 tert-Butyl $(R, E)-(1-p h e n y l n o n-3-e n-5-y n-2-y l) c a r b a m a t e ~ 6 c$}

$780 \mathrm{mg}$ (78\%). Data of the separated major $E$ isomer: brown solid, $\mathrm{mp}=84-85{ }^{\circ} \mathrm{C}$; IR (UATR): 3354, 2219, 1687, $1603 \mathrm{~cm}^{-1} ;{ }^{1} \mathrm{H}$ NMR (300 MHz, $\left.\mathrm{CDCl}_{3}\right): \delta$ 7.33-7.16 (m, 5H), $5.96(\mathrm{dd}, \quad J=15.9 \mathrm{~Hz}, J=5.5 \mathrm{~Hz}, 1 \mathrm{H}), 5.56(\mathrm{dm}, J=15.9 \mathrm{~Hz}, 1 \mathrm{H}), 4.44$ (bs, 2H), $2.82(\mathrm{bd}, J$ $=5.9 \mathrm{~Hz}, 2 \mathrm{H}), 2.25(\mathrm{td}, J=7.0 \mathrm{~Hz}, J=2.1 \mathrm{~Hz}, 2 \mathrm{H}), 1.53(\mathrm{sext}, J=7.2 \mathrm{~Hz}, 2 \mathrm{H}), 1.39(\mathrm{~s}, 9 \mathrm{H})$, $0.97(\mathrm{t}, J=7.3 \mathrm{~Hz}, 3 \mathrm{H}) ;{ }^{13} \mathrm{C} \mathrm{NMR}\left(75 \mathrm{MHz}, \mathrm{CDCl}_{3}\right): \delta 155.1,141.3,137.2,129.6,128.6$, 126.7, 111.0, 91.2, 79.7, 78.5, 53.0, 41.6, 28.5, 22.3, 21.5, 13.7; HRMS (ESI, m/z): Calcd. for $\mathrm{C}_{20} \mathrm{H}_{27} \mathrm{NO}_{2} \mathrm{Na}: 336.1939$, found $[\mathrm{M}+\mathrm{Na}]^{+}: 336.1938$.

\subsubsection{2 (S,E)-2-Hept-1-en-3-yn-1-yl)pyrrolidine-1-carboxylic acid tert-butyl ester 6d}

$740 \mathrm{mg}(100 \%)$, orange solid, $\mathrm{mp}=86-87^{\circ} \mathrm{C}$. Data of the mixture of isomers: IR (UATR): $1688,1594 \mathrm{~cm}^{-1}$; spectroscopic data for the unseparated major $E$ isomer: ${ }^{1} \mathrm{H}$ NMR $\left(300 \mathrm{MHz}, \mathrm{CDCl}_{3}, 50{ }^{\circ} \mathrm{C}\right): \delta 7.42-7.40(\mathrm{~m}, 2 \mathrm{H}), 7.30-7.25(\mathrm{~m}, 3 \mathrm{H}), 6.10(\mathrm{dd}, J=15.7 \mathrm{~Hz}, J=$ $6.2 \mathrm{~Hz}, 1 \mathrm{H}), 5.74(\mathrm{~d}, J=15.9 \mathrm{~Hz}, 1 \mathrm{H}), 4.36(\mathrm{bs}, 1 \mathrm{H}), 3.49-3.32(\mathrm{~m}, 2 \mathrm{H}), 2.08-2.01(\mathrm{~m}, 1 \mathrm{H})$, 1.92-1.79 (m, 2H), 1.77-1.72 (m, 1H), $1.46(\mathrm{~s}, 9 \mathrm{H}) ;{ }^{13} \mathrm{C} \mathrm{NMR}\left(75 \mathrm{MHz}, \mathrm{CDCl}_{3}, 50{ }^{\circ} \mathrm{C}\right): \delta$ 154.6, 144.1, 131.7, 128.4, 128.2, 123.8, 109.8, 89.9, 87.8, 79.6, 58.8, 46.5, 32.2, 28.7, 23.4; HRMS (ESI, m/z): Calcd. for $\mathrm{C}_{19} \mathrm{H}_{23} \mathrm{NO}_{2} \mathrm{Na}: 320.1626$, found $[\mathrm{M}+\mathrm{Na}]^{+}: 320.1622$. 


\subsection{Typical procedure for the preparation of diols 3}

To a solution of enynes 6a-d $(2.0 \mathrm{~m} \mathrm{~mol})$ in $t-\mathrm{BuOH} / \mathrm{H}_{2} \mathrm{O}(1: 1,26 \mathrm{~mL})$ were added successively at $0^{\circ} \mathrm{C}$ AD-mix- $\beta(2.8 \mathrm{~g}, 1.4 \mathrm{~g} / \mathrm{mmol}$ of substrate) , a solution of $0.05 \%(\mathrm{w} / \mathrm{v})$ $\mathrm{OsO}_{4}$ in $t$ - BuOH $(6 \mathrm{~mL}, 0.012 \mathrm{mmol}), \mathrm{NaHCO}_{3}(0.5 \mathrm{~g}, 6.0 \mathrm{mmol})$ and methanesulfonamide $(0.38 \mathrm{~g}, 4 \mathrm{mmol})$. The mixture was stirred vigorously at $0^{\circ} \mathrm{C} 48 \mathrm{~h}$ for $\mathbf{3 a}-\mathbf{c}$ and $24 \mathrm{~h}$ for $\mathbf{3 d}$. The reaction was quenched with $\mathrm{Na}_{2} \mathrm{~S}_{2} \mathrm{O}_{3}(3 \mathrm{~g})$ maintaining vigorous agitation for $30 \mathrm{~min}$ at $0^{\circ} \mathrm{C}$. Diethylether $(20 \mathrm{~mL})$ was added and the mixture was warmed to room temperature under constant agitation. The organic layer was separated and the aqueous layer was extacted with diethylether $(3 \times 40 \mathrm{~mL})$. The combined organic layers were washed with brine, dried over magnesium sulfate and concentrated. Chromatography on silica gel (20\% ethyl acetate in dichloromethane for $\mathbf{3 a}, \mathbf{3 b}$ and $\mathbf{3 d}, 30 \%$ ethyl acetate in dichlorometane for $\mathbf{3 c}$ ) afforded the dihydroxylated derivatives 3a-d as a mixture of diastereomers used in the next step without separation.

\subsection{1 tert-Butyl ((2S)-(1-(benzyloxy)-3,4-dihydroxyoct-5-yn-2-yl)carbamate 3a}

$550 \mathrm{mg}$ (76\%), pale yellow oil. Data of the separated major dihydroxylated product: $[\alpha]_{\mathrm{D}}^{22}=+31.6\left(c\right.$ 1.01, $\left.\mathrm{CH}_{2} \mathrm{Cl}_{2}\right)$; IR (HATR): 3389, 2289, 2232, $1682 \mathrm{~cm}^{-1} ;{ }^{1} \mathrm{H}$ NMR (300 $\left.\mathrm{MHz}, \mathrm{CDCl}_{3}\right): \delta 7.38-7.28(\mathrm{~m}, 5 \mathrm{H}), 5.15(\mathrm{bd}, J=8.7 \mathrm{~Hz}, 1 \mathrm{H}), 4.54(\mathrm{~s}, 2 \mathrm{H}), 4.34(\mathrm{bd}, J=7.5$ $\mathrm{Hz}, 1 \mathrm{H}), 4.12-4.01(\mathrm{~m}, 1 \mathrm{H}), 3.84(\mathrm{bd}, J=6.2 \mathrm{~Hz}, 1 \mathrm{H}), 3.74-3.56(\mathrm{~m}, 2 \mathrm{H}), 3.51(\mathrm{bs}, 1 \mathrm{H})$, 2.94(bs, 1H), 2.22 (qd, $J=7.5 \mathrm{~Hz}, J=1.8 \mathrm{~Hz}, 2 \mathrm{H}), 1.44(\mathrm{~s}, 9 \mathrm{H}), 1.13(\mathrm{t}, J=7.5 \mathrm{~Hz}, 3 \mathrm{H}) ;{ }^{13} \mathrm{C}$ NMR (75 MHz, $\left.\mathrm{CDCl}_{3}\right): \delta 156.3,137.7,128.6,128.0,127,8,89.1,80.0,76.9,75.1,73.5$, 71.2, 64.0, 50.7, 28.5, 13.8, 12.6; HRMS (ESI, m/z): Calcd. for $\mathrm{C}_{20} \mathrm{H}_{29} \mathrm{NO}_{5} \mathrm{Na}$ : 386.1943, found $[\mathrm{M}+\mathrm{Na}]^{+}: 386.1945$.

\subsection{2 tert-Butyl ((2R)-(1-(benzyloxy)-3,4-dihydroxyoct-5-yn-2-yl)carbamate $3 b$}

$520 \mathrm{mg}$ (72\%), pale yellow oil. Data of the separated major dihydroxylated product: $[\alpha]_{\mathrm{D}}^{22}=+3.4\left(c\right.$ 1.35, $\left.\mathrm{CHCl}_{3}\right) ;$ IR (HATR): 3400, 2287, 2233, $1682 \mathrm{~cm}^{-1} ;{ }^{1} \mathrm{H}$ NMR (300 $\left.\mathrm{MHz}, \mathrm{CDCl}_{3}\right): \delta 7.40-7.28(\mathrm{~m}, 5 \mathrm{H}), 5.29(\mathrm{bd}, J=7.4 \mathrm{~Hz}, 1 \mathrm{H}), 4.57$ (AB System, $J_{\mathrm{AB}}=11.7$ $\mathrm{Hz}, 1 \mathrm{H}), 4.51\left(\mathrm{AB}\right.$ System, $\left.J_{\mathrm{AB}}=11.7 \mathrm{~Hz}, 1 \mathrm{H}\right), 4.48-4.43(\mathrm{~m}, 1 \mathrm{H}), 3.95(\mathrm{~d}, J=9.5 \mathrm{~Hz}, 1 \mathrm{H})$, 3.73-3.61 (m, 3H), 3.02 (bs, 2H), 2.24 (qd, $J=7.5 \mathrm{~Hz}, J=2.0 \mathrm{~Hz}, 2 \mathrm{H}), 1.44$ (s, 9H), 1.15 (t, $J$ $=7.5 \mathrm{~Hz}, 3 \mathrm{H}) ;{ }^{13} \mathrm{C} \mathrm{NMR}\left(75 \mathrm{MHz}, \mathrm{CDCl}_{3}\right): \delta 157.1,137.8,128.6,128.0,127,8,88.2,80.7$, 
74.1, 73.7, 69.2, 62.9, 51.6, 28.4, 13.8, 12.6; HRMS (ESI, m/z): Calcd. for $\mathrm{C}_{20} \mathrm{H}_{29} \mathrm{NO}_{5} \mathrm{Na}$ : 386.1943, found $[\mathrm{M}+\mathrm{Na}]^{+}: 386.1945$.

\subsection{3 tert-Butyl ((2R)-3,4-dihydroxy-1-phenylnon-5-yn-2-yl)carbamate 3c}

$520 \mathrm{mg}(75 \%)$. Data of the separated major dihydroxylated product: cream solid, $\mathrm{mp}=$ 108-109 ${ }^{\circ} \mathrm{C} ;[\alpha]_{\mathrm{D}}{ }^{22}=+23.9\left(c 0.78, \mathrm{CH}_{2} \mathrm{Cl}_{2}\right)$; IR (UATR): 3566, 3348, 2227, $1688 \mathrm{~cm}^{-1} ;{ }^{1} \mathrm{H}$ NMR (300 MHz, $\left.\mathrm{CDCl}_{3}\right): \delta 7.35-7.22(\mathrm{~m}, 5 \mathrm{H}), 4.60(\mathrm{bd}, J=8.6 \mathrm{~Hz}, 1 \mathrm{H}), 4.46-4.42(\mathrm{~m}, 1 \mathrm{H})$, 3.96-3.82 (m, 1H), $3.82(\mathrm{bd}, J=4.4 \mathrm{~Hz}, 1 \mathrm{H}), 3.47-3.38(\mathrm{~m}, 1 \mathrm{H}), 3.09$ (dd, $J=14.0 \mathrm{~Hz}, J=$ $4.1 \mathrm{~Hz}, 1 \mathrm{H}), 2.97-2.90(\mathrm{~m}, 1 \mathrm{H}), 2.91(\mathrm{bd}, J=6.0 \mathrm{~Hz}, 1 \mathrm{H}), 2.22(\mathrm{td}, J=7.1 \mathrm{~Hz}, J=2.0 \mathrm{~Hz}$, 2H), 1.55 (sext, $J=7.3 \mathrm{~Hz}, 2 \mathrm{H}), 1.37(\mathrm{~s}, 9 \mathrm{H}), 0.98(\mathrm{t}, J=7.3 \mathrm{~Hz}, 3 \mathrm{H}) ;{ }^{13} \mathrm{C}$ NMR $(75 \mathrm{MHz}$, $\left.\mathrm{CDCl}_{3}\right): \delta 157.1,137.4,129.6,128.8,126.8,87.1,80.7,78.0,76.0,63.1,52.6,36.5,28.4$, 22.1, 20.9, 13.7; HRMS (ESI, m/z): Calcd. for $\mathrm{C}_{20} \mathrm{H}_{29} \mathrm{NO}_{4} \mathrm{Na}: 370.1994$, found $[\mathrm{M}+\mathrm{Na}]^{+}$: 370.1991 .

\subsection{4 (2R)-2-(1,2-Dihydroxyhept-3-yn-1-yl)pyrrolidine-1-carboxylic acid tert-butyl ester 3d}

$520 \mathrm{mg}$ (79\%), pale yellow oil. Data of the separated major dihydroxylated product: $[\alpha]_{\mathrm{D}}^{22}=-100.2$ (c 1.31, $\mathrm{CH}_{2} \mathrm{Cl}_{2}$ ); IR (UATR): 3381, 2243, $1658 \mathrm{~cm}^{-1} ;{ }^{1} \mathrm{H}$ NMR (300 MHz, $\left.\mathrm{CDCl}_{3}\right): \delta 7.47-7.43(\mathrm{~m}, 2 \mathrm{H}), 7.32-7.27(\mathrm{~m}, 3 \mathrm{H}), 5.21(\mathrm{bs}, 1 \mathrm{H}), 4.62-4.53(\mathrm{~m}, 1 \mathrm{H}), 4.13(\mathrm{td}, J$ $=7.6 \mathrm{~Hz}, J=3.6 \mathrm{~Hz}, 1 \mathrm{H}), 3.73-3.67(\mathrm{~m}, 2 \mathrm{H}), 3.57-3.49(\mathrm{~m}, 1 \mathrm{H}), 3.38-3.30(\mathrm{~m}, 1 \mathrm{H}), 2.12-$ $1.80(\mathrm{~m}, 4 \mathrm{H}), 1.46(\mathrm{~s}, 9 \mathrm{H}) ;{ }^{13} \mathrm{C} \mathrm{NMR}\left(75 \mathrm{MHz}, \mathrm{CDCl}_{3}\right): \delta 158.4,132.0,128.5,128.3,122.7$, 88.2, 85.3, 81.2, 78.2, 64.5, 59.6, 47.7, 29.2, 28.5, 24.3; HRMS (ESI, m/z): Calcd. for $\mathrm{C}_{19} \mathrm{H}_{25} \mathrm{NO}_{4} \mathrm{Na}: 354.1681$, found $[\mathrm{M}+\mathrm{Na}]^{+}: 354.1681$.

\subsection{Typical procedure for the synthesis of furans 1}

To a degassed solution of dihydroxylated derivatives 3a-c $(0.6 \mathrm{mmol})$ in $1,2-$ dichloroethane $(4 \mathrm{~mL})$ was added under argon atmosphere $0.4 \mathrm{~mL}$ of a freshly prepared solution of $\mathrm{PPh}_{3} \mathrm{AuCl}(14.8 \mathrm{mg}, 0.03 \mathrm{mmol})$ and $\mathrm{AgSbF}_{6}(10.3 \mathrm{mg}, 0.03 \mathrm{mmol})$ in $2 \mathrm{~mL}$ degassed 1,2- dichloroethane. After stirring for $1 \mathrm{~h}$ at room temperature, the reaction mixture was concentrated and purified by silica gel chromatography eluting with dichloromethane to afford the 2,5-disubstitued furans 1a-c. 


\subsection{1 tert-Butyl (S)-(2-(benzyloxy)-1-(5-ethylfuran-2-yl)ethyl)carbamate 1a}

$184 \mathrm{mg}(89 \%)$, colorless oil. $[\alpha]_{\mathrm{D}}^{22}=-35.1$ (c 1.00, $\left.\mathrm{CH}_{2} \mathrm{Cl}_{2}\right), 94 \%$ ee; IR (UATR): 3336, $1705 \mathrm{~cm}^{-1} ;{ }^{1} \mathrm{H}$ NMR $\left(300 \mathrm{MHz}, \mathrm{CDCl}_{3}\right): \delta 7.38-7.24(\mathrm{~m}, 5 \mathrm{H}), 6.11(\mathrm{~d}, J=3.1 \mathrm{~Hz}, 1 \mathrm{H}), 5.89$ $(\mathrm{dt}, J=3.1 \mathrm{~Hz}, J=0.9 \mathrm{~Hz}, 1 \mathrm{H}), 5.08$ (bs, $1 \mathrm{H}), 4.93(\mathrm{bs}, 1 \mathrm{H}), 4.55$ (AB System, $J_{\mathrm{AB}}=12.1$ $\mathrm{Hz}, 1 \mathrm{H}), 4.49\left(\mathrm{AB}\right.$ System, $\left.J_{\mathrm{AB}}=12.1 \mathrm{~Hz}, 1 \mathrm{H}\right), 3.76(\mathrm{dd}, J=9.7 \mathrm{~Hz}, J=4.9 \mathrm{~Hz}, 1 \mathrm{H}), 3.70$ $(\mathrm{dd}, J=9.7 \mathrm{~Hz}, J=5.0 \mathrm{~Hz}, 1 \mathrm{H}), 2.60(\mathrm{q}, J=7.5 \mathrm{~Hz}, 2 \mathrm{H}), 1.44(\mathrm{~s}, 9 \mathrm{H}), 1.20(\mathrm{t}, J=7.5 \mathrm{~Hz}$, $3 \mathrm{H}) ;{ }^{13} \mathrm{C}$ NMR $\left(75 \mathrm{MHz} \mathrm{CDCl}_{3}\right): \delta 157.3,155.4,151.1,138.1,128.5,127.8,127.7,107.2$, 104.7, 79.8, 73.2, 71.0, 48.9, 28.5, 21.5, 12.2; HRMS (ESI, m/z): Calcd. for $\mathrm{C}_{20} \mathrm{H}_{27} \mathrm{NO}_{4} \mathrm{Na}$ : 368.1832, found $[\mathrm{M}+\mathrm{Na}]^{+}:$368.1833; HPLC analysis (Chiralpak IA column, heptane/2propanol $=95 / 5$, flow rate $=1 \mathrm{~mL} / \mathrm{min}$, wavelength $=230 \mathrm{~nm}$ ): $\mathrm{Rt}=7.42$ (major) and 8.22 (minor).

\subsection{2 tert-Butyl (R)-(2-(benzyloxy)-1-(5-ethylfuran-2-yl)ethyl)carbamate 1b}

$184 \mathrm{mg}(89 \%)$, colorless oil. $[\alpha]_{\mathrm{D}}{ }^{22}=+33.4\left(c 0.99, \mathrm{CH}_{2} \mathrm{Cl}_{2}\right), 93 \%$ ee; spectral data and HRMS are identical with those reportated for 1a; HPLC analysis (Chiralpak IA column, heptane $/ 2$-propanol $=95 / 5$, flow rate $=1 \mathrm{ml} / \mathrm{min}$, wavelength $=230 \mathrm{~nm}): \mathrm{Rt}=7.40$ (minor) and 8.19 (major).

\subsection{3 tert-Butyl (R)-(2-phenyl-1-(5-propylfuran-2-yl)ethyl)carbamate 1c}

$166 \mathrm{mg}(84 \%)$, colorless oil. $[\alpha]_{\mathrm{D}}^{22}=+43.4$ (c 1.07, $\left.\mathrm{CH}_{2} \mathrm{Cl}_{2}\right), \geq 99 \%$ ee; IR (UATR): 3343, $1701 \mathrm{~cm}^{-1} ;{ }^{1} \mathrm{H}$ NMR (300 MHz, $\left.\mathrm{CDCl}_{3}\right): \delta 7.25-7.17(\mathrm{~m}, 3 \mathrm{H}), 7.06-7.03(\mathrm{~m}, 2 \mathrm{H}), 5.88(\mathrm{~d}, J=$ $3.0 \mathrm{~Hz}, 1 \mathrm{H}), 5.84(\mathrm{~d}, J=3.0 \mathrm{~Hz}, 1 \mathrm{H}), 4.93$ (bs, 1H), 4.82 (bs, 1H), 3.09 (bd, $J=6.6 \mathrm{~Hz}, 2 \mathrm{H})$, $2.57(\mathrm{t}, J=7.4 \mathrm{~Hz}, 2 \mathrm{H}), 1.65(\mathrm{sext}, J=7.4 \mathrm{~Hz}, 2 \mathrm{H}), 1.40(\mathrm{~s}, 9 \mathrm{H}), 0.96(\mathrm{t}, J=7.4 \mathrm{~Hz}, 3 \mathrm{H}) ;{ }^{13} \mathrm{C}$ NMR (75 MHz, $\left.\mathrm{CDCl}_{3}\right): \delta 155.7,155.1,151.9,137.5,129.6,128.3,126.5,107.0,105.5,79.7$, 50.3, 40.8, 30.2, 28.5, 21.6, 13.8; HRMS (ESI, m/z): Calcd. for $\mathrm{C}_{20} \mathrm{H}_{27} \mathrm{NO}_{3} \mathrm{Na}$ : 352.1889, found $[\mathrm{M}+\mathrm{Na}]^{+}$: 352.1887; HPLC analysis (Chiralpak IA column, heptane/2-propanol = 99/1, flow rate $=1 \mathrm{~mL} / \mathrm{min}$, wavelength $=220 \mathrm{~nm}): \mathrm{Rt}=10.08$. 


\subsection{Typical procedure for the synthesis of iodofurans 2}

To a degassed solution of dihydroxylated derivatives 3a-d $(0.6 \mathrm{mmol})$ in 1,2dichloroethane $(4 \mathrm{~mL})$ were added successively under argon atmosphere NIS $(0.16 \mathrm{~g}, 0.72$ $\mathrm{mmol})$ and $0.4 \mathrm{~mL}$ of a freshly prepared solution of $\mathrm{PPh}_{3} \mathrm{AuCl}(14.8 \mathrm{mg}, 0.03 \mathrm{mmol})$ and $\mathrm{AgSbF}_{6}(10.3 \mathrm{mg}, 0.03 \mathrm{mmol})$ in $2 \mathrm{~mL}$ degassed 1,2- dichloroethane. After stirring for $1 \mathrm{~h}$ at room temperature, $20 \% \mathrm{Na}_{2} \mathrm{~S}_{2} \mathrm{O}_{3}(5 \mathrm{~mL})$ was added and the mixture was extracted with diethylether $(3 \times 10 \mathrm{~mL})$. The combined organic extracts were washed with brine, dried over magnesium sulfate and concentrated. Chromatography on silica gel eluting with dichloromethane afforded the 2,5-disubstituted 3-iodofurans 2a-d.

\subsection{1 tert-Butyl (S)-(2-(benzyloxy)-1-(5-ethyl-4-iodofuran-2-yl)ethyl)carbamate 2a}

$175 \mathrm{mg}(62 \%)$, pale yellow oil. $[\alpha]_{\mathrm{D}}{ }^{22}=-36.5$ (c 1.02, $\left.\mathrm{CH}_{2} \mathrm{Cl}_{2}\right), 96 \%$ ee; IR (UATR): 3332, $1704 \mathrm{~cm}^{-1} ;{ }^{1} \mathrm{H}$ NMR $\left(300 \mathrm{MHz}, \mathrm{CDCl}_{3}\right): \delta$ 7.37-7.24 (m, 5H), $6.20(\mathrm{~s}, 1 \mathrm{H}), 511$ (bs, 1H), $4.91(\mathrm{bs}, 1 \mathrm{H}), 4.55\left(\mathrm{AB}\right.$ System, $\left.J_{\mathrm{AB}}=12.1 \mathrm{~Hz}, 1 \mathrm{H}\right), 4.48\left(\mathrm{AB}\right.$ System, $\left.J_{\mathrm{AB}}=12.1 \mathrm{~Hz}, 1 \mathrm{H}\right)$, $3.75(\mathrm{dd}, J=9.6 \mathrm{~Hz}, J=4.4 \mathrm{~Hz}, 1 \mathrm{H}), 3.68(\mathrm{dd}, J=9.6 \mathrm{~Hz}, J=4.7 \mathrm{~Hz}, 1 \mathrm{H}), 2.64(\mathrm{q}, J=7.6$ $\mathrm{Hz}, 2 \mathrm{H}), 1.45$ (s, 9H), $1.17(\mathrm{t}, J=7.6 \mathrm{~Hz}, 3 \mathrm{H}) ;{ }^{13} \mathrm{C} \mathrm{NMR}\left(75 \mathrm{MHz}, \mathrm{CDCl}_{3}\right): \delta 157.1,155.3$, 152.8, 137.9, 128.6, 127.9, 127.8, 114.1, 80.1, 73.3, 70.6, 61.7, 48.8, 28.5, 21.1, 12.6; HRMS (ESI, m/z): Calcd. for $\mathrm{C}_{20} \mathrm{H}_{26} \mathrm{NO}_{4} \mathrm{INa}$ : 494.0804, found [M+Na] $]^{+}$: 494.0806; HPLC analysis (Chiralpak IA column, heptane $/ 2$-propanol $=97 / 3$, flow rate $=1 \mathrm{ml} / \mathrm{min}$, wavelength $=230$ $\mathrm{nm}$ ): Rt $=9.06$ (major) and 9.89 (minor).

\subsection{2 tert-Butyl (R)-(2-(benzyloxy)-1-(5-ethyl-4-iodofuran-2-yl)ethyl)carbamate $2 b$}

$175 \mathrm{mg}(62 \%)$, pale yellow oil. $[\alpha]_{\mathrm{D}}^{22}=+36.3\left(c 1.03, \mathrm{CH}_{2} \mathrm{Cl}_{2}\right), 94 \%$ ee; spectral data and HRMS are identical with those reportated for $\mathbf{2 a}$; HPLC analysis (Chiralpak IA column, heptane $/ 2$-propanol $=97 / 3$, flow rate $=1 \mathrm{ml} / \mathrm{min}$, wavelength $=230 \mathrm{~nm}): \mathrm{Rt}=9.09$ (minor) and 9.91 (major).

\subsection{3 tert-Butyl (R)-(1-(4-iodo-5-propylfuran-2-yl)-2-phenylethyl)carbamate 2c}

$153 \mathrm{mg}(56 \%)$, white solid, $\mathrm{mp}=79-80{ }^{\circ} \mathrm{C} ;[\alpha]_{\mathrm{D}}^{22}=+38.3\left(c 1.08, \mathrm{CH}_{2} \mathrm{Cl}_{2}\right.$ ); IR (UATR): 3316, $1685 \mathrm{~cm}^{-1} ;{ }^{1} \mathrm{H}$ NMR (300 $\left.\mathrm{MHz}, \mathrm{CDCl}_{3}\right): \delta 7.28-7.17(\mathrm{~m}, 3 \mathrm{H}), 7.06-7.03(\mathrm{~m}, 2 \mathrm{H}), 6.02$ (s, 1H), 4.95 (bs, 1H), 4.76 (bs, 1H), 3.07 (bd, $J=6.5 \mathrm{~Hz}, 2 \mathrm{H}), 2.62$ (t, $J=7.4 \mathrm{~Hz}, 2 \mathrm{H}), 1.65$ 
(sext, $J=7.4 \mathrm{~Hz}, 2 \mathrm{H}), 1.40(\mathrm{~s}, 9 \mathrm{H}), 0.95(\mathrm{t}, J=7.4 \mathrm{~Hz}, 3 \mathrm{H}) ;{ }^{13} \mathrm{C} \mathrm{NMR}\left(75 \mathrm{MHz}, \mathrm{CDCl}_{3}\right): \delta$ 156.0, 155.0, 153.7, 136.9, 129.5, 128.5, 126.8, 113.9, 80.0, 62.7, 49.9, 40.4, 29.4, 28.4, 21.8, 13.7; HRMS (ESI, m/z): Calcd. for $\mathrm{C}_{20} \mathrm{H}_{26} \mathrm{NO}_{3} \mathrm{INa}$ : 478.0855, found [M+Na] $]^{+}$: 478.0854 .

\subsection{4 (S)-2 -(4-Iodo-5-phenylfuran-2-yl)pyrrolidine-1-carboxylic acid tert-butyl ester 2d}

$121 \mathrm{mg}(46 \%)$, yellow solid, $\mathrm{mp}=71-72{ }^{\circ} \mathrm{C} ;[\alpha]_{\mathrm{D}}^{22}=-101.0\left(c 1.04, \mathrm{CH}_{2} \mathrm{Cl}_{2}\right)$; IR (UATR): $1690 \mathrm{~cm}^{-1} ;{ }^{1} \mathrm{H}$ NMR $\left(500 \mathrm{MHz}, \mathrm{CDCl}_{3}, 55{ }^{\circ} \mathrm{C}\right): \delta 7.92(\mathrm{~d}, J=7.3 \mathrm{~Hz}, 2 \mathrm{H}), 7.39(\mathrm{t}, J=7.7$ $\mathrm{Hz}, 2 \mathrm{H}), 7.30$ (t, $J=7.4 \mathrm{~Hz}, 1 \mathrm{H}), 6.29$ (s, 1H), 4.90 (bs, 1H), 3.59-3.41 (m, 2H), 2.23-2.13 $(\mathrm{m}, 1 \mathrm{H}), 2.12-2.00(\mathrm{~m}, 2 \mathrm{H}), 1.96-1.88(\mathrm{~m}, 1 \mathrm{H}), 1.40(\mathrm{~s}, 9 \mathrm{H}) ;{ }^{13} \mathrm{C} \mathrm{NMR}\left(125 \mathrm{MHz}, \mathrm{CDCl}_{3}, 55\right.$ $\left.{ }^{\circ} \mathrm{C}\right): \delta 157.1,154.5,150.8,130.6,128.5,128.1,126.3,116.5,79.9,61.2,54.8,46.5,32.4$, 28.6, 23.9; HRMS (ESI, m/z): Calcd. for $\mathrm{C}_{19} \mathrm{H}_{22} \mathrm{NO}_{3} \mathrm{INa}$ : 462.0542, found $[\mathrm{M}+\mathrm{Na}]^{+}$: 462.0540 .

\subsection{Typical procedure for the Suzuki-Miyaura coupling of iodofurans 2 with 4- substituted phenylboronic acid}

To a degassed solution of iodofurans $\mathbf{2 a}, \mathbf{2 c}$ or $\mathbf{2 d}(0.25 \mathrm{mmol})$ and the appropriate aryl boronic acid $(0.375 \mathrm{mmol})$ in toluene $(2.5 \mathrm{~mL})$ were added successively under argon atmosphere $\mathrm{K}_{3} \mathrm{PO}_{4}(106.0 \mathrm{mg}, 0.50 \mathrm{mmol})$, S-Phos (10.3 $\left.\mathrm{mg}, 0.025 \mathrm{mmol}, 10 \mathrm{~mol} \%\right)$ and $\mathrm{Pd}(\mathrm{OAc})_{2}(2.8 \mathrm{mg}, 0.0125 \mathrm{mmol}, 5 \mathrm{~mol} \%)$. The resulting mixture was heated to $80^{\circ} \mathrm{C}$ for $3 \mathrm{~h}$. After cooling, the mixture was diluted with diethylether, filtered over a Celite ${ }^{\circledR}$ plug, wich was washed with diethylether. After removal of solvents in vaccuo, the crude product was purified by silica gel chromatography eluting with $20 \%$ diethylether in petoleum ether for $\mathbf{7 , 8}$ and $25 \%$ ethyl acetate in cyclohexane for 9.

\subsection{1 tert-Butyl (S)-(2-(benzyloxy)-1-(5-ethyl-4-(4-fluorophenyl)furan-2-}

\section{yl)ethyl)carbamate 7}

$96 \mathrm{mg}(87 \%)$, colorless oil; $[\alpha]_{\mathrm{D}}{ }^{22}=-36.4\left(c 0.99, \mathrm{CH}_{2} \mathrm{Cl}_{2}\right), 96 \% e e$; IR (UATR): 3336, $1711 \mathrm{~cm}^{-1} ;{ }^{1} \mathrm{H}$ NMR $\left(300 \mathrm{MHz}, \mathrm{CDCl}_{3}\right): \delta 7.36-7.24(\mathrm{~m}, 7 \mathrm{H}), 7.10-7.02(\mathrm{~m}, 2 \mathrm{H}), 6.28(\mathrm{~s}$, $1 \mathrm{H}), 5.14$ (bs, 1H), 4.97 (bs, 1H), 4.58 (AB System, $\left.J_{\mathrm{AB}}=12.2 \mathrm{~Hz}, 1 \mathrm{H}\right), 4.52$ (AB System, $\left.J_{\mathrm{AB}}=12.2 \mathrm{~Hz}, 1 \mathrm{H}\right), 3.81(\mathrm{dd}, J=9.7 \mathrm{~Hz}, J=4.6 \mathrm{~Hz}, 1 \mathrm{H}), 3.75(\mathrm{dd}, J=9.7 \mathrm{~Hz}, J=4.8 \mathrm{~Hz}$, $1 \mathrm{H}), 272(\mathrm{q}, J=7.5 \mathrm{~Hz}, 2 \mathrm{H}), 1.46(\mathrm{~s}, 9 \mathrm{H}), 1.24(\mathrm{t}, J=7.5 \mathrm{~Hz}, 3 \mathrm{H}) ;{ }^{13} \mathrm{C} \mathrm{NMR}(75 \mathrm{MHz}$, 
$\left.\mathrm{CDCl}_{3}\right): \delta 161.7\left(J_{\mathrm{CF}}^{1}=245.3 \mathrm{~Hz}\right), 155.4,152.1,151.0,138.1,130.4\left(J_{\mathrm{CF}}^{4}=3.3 \mathrm{~Hz}\right), 129.3$ $\left(J^{3} \mathrm{CF}=7.9 \mathrm{~Hz}\right), 128.5,127.8,127.7,120.2,115.5\left(J^{2} \mathrm{CF}=21.4 \mathrm{~Hz}\right), 108.3,80.0,73.3,70.9$, 48.9, 28.5, 20.4, 13.1; HRMS (ESI, m/z): Calcd. for $\mathrm{C}_{26} \mathrm{H}_{30} \mathrm{NO}_{4} \mathrm{FNa}$ : 462.2057, found $[\mathrm{M}+\mathrm{Na}]^{+}$: 462.2059; HPLC analysis (Chiralpak IA column, heptane/2-propanol = 95/5, flow rate $=1 \mathrm{~mL} / \mathrm{min}$, wavelength $=220 \mathrm{~nm}$ ): $\mathrm{Rt}=10.01$ (minor) and 10.70 (major).

\subsection{2 tert-Butyl (R)-(1-(4-(4-fluorophenyl)-5-propylfuran-2-yl)-2-phenylethyl)carbamate} 8

$103 \mathrm{mg}(95 \%)$, colorless oil; $[\alpha]_{\mathrm{D}}^{22}=+36.6\left(c 1.00, \mathrm{CH}_{2} \mathrm{Cl}_{2}\right), \geq 99 \%$ ee; IR (UATR): 3345 , $1700 \mathrm{~cm}^{-1} ;{ }^{1} \mathrm{H}$ NMR $\left(300 \mathrm{MHz}, \mathrm{CDCl}_{3}\right): \delta$ 7.26-7.16 (m, 5H), 7.10-7.01 (m, 4H), $6.07(\mathrm{~s}$, 1H), 4.99 (bs, 1H), 4.85 (bs, 1H), 3.13 (bd, $J=6.5 \mathrm{~Hz}, 2 \mathrm{H}), 2.69$ (t, $J=7.5 \mathrm{~Hz}, 2 \mathrm{H}), 1.71$ (sext, $J=7.4 \mathrm{~Hz}, 2 \mathrm{H}), 1.41(\mathrm{~s}, 9 \mathrm{H}), 0.96(\mathrm{t}, J=7.4 \mathrm{~Hz}, 3 \mathrm{H}) ;{ }^{13} \mathrm{C} \mathrm{NMR}\left(75 \mathrm{MHz}, \mathrm{CDCl}_{3}\right): \delta$ $161.7\left(J_{\mathrm{CF}}^{1}=245.5 \mathrm{~Hz}\right), 155.1,151.8,151.0,137.3,130.4\left(J_{\mathrm{CF}}^{4}=3.2 \mathrm{~Hz}\right), 129.6,129.3\left(J^{3} \mathrm{CF}\right.$ $=7.9 \mathrm{~Hz}), 128.4,126.7,120.7,115.5\left(J_{\mathrm{CF}}^{2}=21.4 \mathrm{~Hz}\right), 108.1,79.9,50.1,40.7,28.9,28.5$, 22.1, 14.0; HRMS (ESI, m/z): Calcd. for $\mathrm{C}_{26} \mathrm{H}_{30} \mathrm{NO}_{3} \mathrm{FNa}$ : 446.2107, found $[\mathrm{M}+\mathrm{Na}]^{+}$: 446.2107; HPLC analysis (Chiralpak IA column, heptane/2-propanol $=98 / 2$, flow rate $=1$ $\mathrm{mL} / \mathrm{min}$, wavelength $=220 \mathrm{~nm}): \mathrm{Rt}=10.65$.

\subsection{3 (S)-2-(4-(4-((tert-Butoxycarbonyl)amino)phenyl)-5-phenylfuran-2-yl)pyrrolidine-1- carboxylic acid tert-butyl ester 9}

$119 \mathrm{mg}(94 \%)$, white solid, $\mathrm{mp}=220-221{ }^{\circ} \mathrm{C} ;[\alpha]_{\mathrm{D}}{ }^{22}=-86.3\left(c 0.91, \mathrm{CH}_{2} \mathrm{Cl}_{2}\right), 83 \%$ ee; IR (UATR): $3247,1726,1672 \mathrm{~cm}^{-1}$; ${ }^{1} \mathrm{H}$ NMR (300 MHz, DMSO, $80{ }^{\circ} \mathrm{C}$ ): $\delta 9.13$ (bs, $\left.1 \mathrm{H}\right), 7.48$ $7.43(\mathrm{~m}, 4 \mathrm{H}), 7.35-7.30(\mathrm{~m}, 2 \mathrm{H}), 7.27-7.22(\mathrm{~m}, 3 \mathrm{H}), 6.32(\mathrm{~s}, 1 \mathrm{H}), 4.88(\mathrm{dd}, J=7.7 \mathrm{~Hz}, J=2.5$ $\mathrm{Hz}, 1 \mathrm{H}), 3.52-3.36(\mathrm{~m}, 2 \mathrm{H}), 2.29-2.16(\mathrm{~m}, 1 \mathrm{H}), 2.11-1.86(\mathrm{~m}, 3 \mathrm{H}), 1.50(\mathrm{~s}, 9 \mathrm{H}), 1.36(\mathrm{~s}, 9 \mathrm{H})$; ${ }^{13} \mathrm{C}$ NMR $\left(75 \mathrm{MHz}\right.$, DMSO, $\left.80{ }^{\circ} \mathrm{C}\right): \delta 155.1,153.1,152.4,145.5,138.4,130.6,128.1,128.0$, 127.1, 127.0, 125.1, 122.2, 118.2, 109.1, 78.8, 78.2, 53.9, 45.7, 31.1, 27.8, 27.7, 23.0; HRMS (ESI, m/z): Calcd. for $\mathrm{C}_{30} \mathrm{H}_{36} \mathrm{~N}_{2} \mathrm{O}_{5} \mathrm{Na}$ : 527.2522, found [M+Na] $]^{+}$: 527.2519; HPLC analysis (Chiralpak IA column, heptane $/ 2$-propanol $=90 / 10$, flow rate $=1 \mathrm{~mL} / \mathrm{min}$, wavelength $=250$ $\mathrm{nm}): \mathrm{Rt}=7.51$ (minor) and 10.27 (major).

4.8 (R,E)-3-(5-(1-((tert-butoxycarbonyl)amino)-2-phenylethyl)-2-propylfuran-3-yl)acrylic acid methyl ester 10 
To a degassed solution of iodofuran $2 \mathbf{c}(114 \mathrm{mg}, 0.25 \mathrm{mmol})$ in dry DMF (2 mL) were added successively under argon atmosphere methyl acrylate ( $45 \mu 1,0.5 \mathrm{mmol})$, triethylamine (70 $\mu 1,0.5 \mathrm{mmol})$ and $\mathrm{Pd}\left(\mathrm{PPh}_{3}\right)_{4}(29 \mathrm{mg}, 0.025 \mathrm{mmol}, 10 \mathrm{~mol} \%)$. The mixture was heated to $80{ }^{\circ} \mathrm{C}$ for $2 \mathrm{~h}$. After cooling, $\mathrm{H}_{2} \mathrm{O}(3 \mathrm{~mL})$ was added and the mixture was extracted with diethylether $(3 \times 5 \mathrm{~mL})$. The combined organic extracts were washed with brine, dried over magnesium sulfate and concentrated. Chromatography on silica gel eluting with $2 \%$ diethylether in dichloromethane afforded the pure product $\mathbf{1 0}$ as a yellow solid: $62 \mathrm{mg}(60 \%)$, $\mathrm{mp}=104-105{ }^{\circ} \mathrm{C} ;[\alpha]_{\mathrm{D}}^{22}=+31.7\left(c 0.94, \mathrm{CH}_{2} \mathrm{Cl}_{2}\right), 99 \%$ ee; IR (UATR): 3343, 1714, 1688, $1634 \mathrm{~cm}^{-1} ;{ }^{1} \mathrm{H}$ NMR $\left(300 \mathrm{MHz}, \mathrm{CDCl}_{3}\right): \delta 7.48(\mathrm{~d}, J=15.7 \mathrm{~Hz}, 1 \mathrm{H}), 7.27-7.17(\mathrm{~m}, 3 \mathrm{H})$, 7.06-7.04 (m, 2H), 6.12 (s, 1H), 5.95 (d, $J=15.7 \mathrm{~Hz}, 1 \mathrm{H}), 4.97$ (bs, 1H), 4.80 (bs, 1H), 3.76 (s, 3H), 3.09 (bd, $J=6.6 \mathrm{~Hz}, 2 \mathrm{H}), 2.69$ (t, $J=7.4 \mathrm{~Hz}, 2 \mathrm{H}), 1.68$ (sext, $J=7.4 \mathrm{~Hz}, 2 \mathrm{H}), 1.41$ (s, 9H), 0.95 (t, $J=7.4 \mathrm{~Hz}, 3 \mathrm{H}) ;{ }^{13} \mathrm{C} \mathrm{NMR}\left(75 \mathrm{MHz}, \mathrm{CDCl}_{3}\right): \delta 167.9,158.0,155.0,153.6$, 136.9, 135.4, 129.5, 128.5, 126.8, 118.1, 115.8, 104.4, 80.1, 51.6, 50.0, 40.4, 28.5, 28.3, 22.1, 13.8; HRMS (ESI, m/z): Calcd. for $\mathrm{C}_{24} \mathrm{H}_{31} \mathrm{NO}_{5} \mathrm{Na}$ : 436.2100, found $[\mathrm{M}+\mathrm{Na}]^{+}: 436.2101$; HPLC analysis (Chiralpak IA column, heptane/2-propanol $=98 / 2$, flow rate $=1 \mathrm{~mL} / \mathrm{min}$, wavelength $=290 \mathrm{~nm}$ ): Rt =20.29 (major), 27.17 (minor).

\section{9 (R)-(2-Phenyl-1-(4-(phenylethynyl)-5-propylfuran-2-yl)ethyl)carbamic acid tert-butyl ester 11}

To a degassed solution of iodofuran $2 \mathrm{c}(114 \mathrm{mg}, 0.25 \mathrm{mmol})$ in triethylamine $(2 \mathrm{~mL})$ were added under argon atmoshere $\mathrm{PdCl}_{2}\left(\mathrm{PPh}_{3}\right)_{2}(1.8 \mathrm{mg}, 0.0025 \mathrm{mmol}, 1 \mathrm{~mol} \%)$ and $\mathrm{CuI}(1.0 \mathrm{mg}$, $0.005 \mathrm{mmol}, 2 \mathrm{~mol} \%$ ). The reaction mixture was stirred for $15 \mathrm{~min}$ at room temperature. Then a solution of phenylacetylene $(69 \mu 1,0.63 \mathrm{mmol})$ in triethylamine $(0.4 \mathrm{~mL})$ was added dropwise and the mixture was stirred for $4 \mathrm{~h}$ at room temperature. Subsequently, the mixture was diluted with diethylether $(10 \mathrm{~mL})$ and washed with brine. The organic extract was separated, dried over magnesium sulfate and concentated. Chromatography on silica gel eluting with $20 \%$ diethylether in petroleum ether afforded the pure product $\mathbf{1 1}$ as a pale yellow solid: $101 \mathrm{mg}(94 \%), \mathrm{mp}=96-97{ }^{\circ} \mathrm{C} ;[\alpha]_{\mathrm{D}}^{22}=+34.2\left(c 1.04, \mathrm{CH}_{2} \mathrm{Cl}_{2}\right), \geq 99 \%$ ee; IR (UATR): 3389, 2223, $1687 \mathrm{~cm}^{-1} ;{ }^{1} \mathrm{H}$ NMR (300 MHz, $\left.\mathrm{CDCl}_{3}\right): \delta 7.45-7.43$ (m, 2H), 7.35$7.17(\mathrm{~m}, 10 \mathrm{H}), 7.08-7.05(\mathrm{~m}, 2 \mathrm{H}), 6.06(\mathrm{~s}, 1 \mathrm{H}), 4.96(\mathrm{bs}, 1 \mathrm{H}), 4.79$ (bs, 1H), 3.10 (bd, $J=6.7$ $\mathrm{Hz}, 2 \mathrm{H}), 2.74$ (t, $J=7.4 \mathrm{~Hz}, 2 \mathrm{H}), 1.74$ (sext, $J=7.4 \mathrm{~Hz}, 2 \mathrm{H}), 1.41$ (s, 9H), 0.99 (t, $J=7.4 \mathrm{~Hz}$, $3 \mathrm{H}) ;{ }^{13} \mathrm{C}$ NMR $\left(75 \mathrm{MHz}, \mathrm{CDCl}_{3}\right): \delta 159.5,155.0,152.1,137.0,131.4,129.5,128.5,128.4$, 128.0, 126.7, 123.8, 109.4, 103.7, 91.8, 81.9, 80.0, 50.0, 40.5, 29.3, 28.5, 21.6, 13.9; HRMS 
(ESI, m/z): Calcd. for $\mathrm{C}_{28} \mathrm{H}_{31} \mathrm{NO}_{3} \mathrm{Na}$ : 452.2202, found [M+Na $]^{+}:$452.2205; HPLC analysis (Chiralpak IA column, heptane $/ 2$-propanol $=98 / 2$, flow rate $=1 \mathrm{~mL} / \mathrm{min}$, wavelength $=280$ $\mathrm{nm}): \mathrm{Rt}=10.63$.

\subsection{0 (S)-2-(4-((tert-butoxycarbonyl)amino)-5-phenylfuran-2-yl)pyrrolidine-1-carboxylic acid tert-butyl ester 12}

To a degassed mixture of iodofuran $\mathbf{2 d}(110 \mathrm{mg}, 0.25 \mathrm{mmol})$, tert-butyl carbamate (35 $\mathrm{mg}, 0.3 \mathrm{mmol})$, cesium carbonate (122 mg, $0.37 \mathrm{mmol})$ and $\mathrm{CuI}$ (9.5 mg, $0.05 \mathrm{mmol}, 20$ mol\%) in dry toluene $(2.5 \mathrm{~mL})$, was added under argon atmosphere N,N'-DMEDA (11 $\mu 1,0.1$ $\mathrm{mmol}, 40 \mathrm{~mol} \%$ ). The mixture was heated to $90{ }^{\circ} \mathrm{C}$ for $18 \mathrm{~h}$. After cooling, the mixture was diluted with dichloromethane, filtered over a Celite plug, which was washed with dichloromethane. After removal of solvents in vaccuo, the crude product was purified by silica gel chromatography eluting with 5\% ethyl acetate in dichloromethane to afford the pure product 12 as a colorless oil: $22 \mathrm{mg}(20 \%)$; $[\alpha]_{\mathrm{D}}^{22}=-89.0\left(\right.$ c $\left.1.23, \mathrm{CH}_{2} \mathrm{Cl}_{2}\right), 97 \%$ ee; IR (UATR): 3291, 1720, $1679 \mathrm{~cm}^{-1}$; ${ }^{1} \mathrm{H}$ NMR (300 MHz, DMSO, $80{ }^{\circ} \mathrm{C}$ ): $\delta 8.38$ (bs, $\left.1 \mathrm{H}\right), 7.61$ $(\mathrm{d}, J=8.3 \mathrm{~Hz}, 2 \mathrm{H}), 7.40(\mathrm{t}, J=7.7 \mathrm{~Hz}, 2 \mathrm{H}), 7.24(\mathrm{t}, J=7.4 \mathrm{~Hz}, 1 \mathrm{H}), 6.28(\mathrm{~s}, 1 \mathrm{H}), 4.83(\mathrm{dd}, J$ $=7.8 \mathrm{~Hz}, J=2.3 \mathrm{~Hz}, 1 \mathrm{H}), 3.49-3.35(\mathrm{~m}, 2 \mathrm{H}), 2.26-2.13(\mathrm{~m}, 1 \mathrm{H}), 2.06-1.88(\mathrm{~m}, 3 \mathrm{H}), 1.43(\mathrm{~s}$, 9H), 1.37 (s, 9H); ${ }^{13} \mathrm{C}$ NMR (75 MHz, DMSO, $\left.80{ }^{\circ} \mathrm{C}\right): \delta 153.6,153.4,153.1,141.4,130.0$, 128.0, 126.3, 123.8, 121.0, 107.2, 78.6, 78.3, 54.0, 45.7, 31.0, 27.7, 22.9; HRMS (ESI, m/z): Calcd. for $\mathrm{C}_{24} \mathrm{H}_{32} \mathrm{~N}_{2} \mathrm{O}_{5} \mathrm{Na}$ : 451.2209, found $[\mathrm{M}+\mathrm{Na}]^{+}$: 451.2207; HPLC analysis (Chiralpak IA column, heptane $/ 2$-propanol $=95 / 5$, flow rate $=1 \mathrm{~mL} / \mathrm{min}$, wavelength $=291 \mathrm{~nm}$ ): Rt $=$ 10.55 (major) and 17.67 (minor).

\subsection{Typical procedure for $O$-debenzylation}

To a solution of furans $\mathbf{1 a}$ or $7(0.2 \mathrm{mmol})$ in ethanol $(2.4 \mathrm{~mL})$ and cyclohexene $(1.2 \mathrm{~mL})$ was added $20 \%$ palladium hydroxyde on carbon (25\% catalyst / substrate by weight). The suspension was stirred under reflux for $18 \mathrm{~h}$. After cooling, the catalyst was filtered over a Celite plug, washed with dichloromethane and the volatiles were removed by evaporation in vacuo. The residue was purified by silica gel chromatography eluting with a mixture of cyclohexane / ethyl acetate / methanol (75 / 25 /1). 


\subsection{1 (S)-(1-(5-ethylfuran-2-yl)-2-hydroxyethyl)carbamic acid tert-butyl ester 13}

$47 \mathrm{mg}(93 \%)$, colorless oil; $[\alpha]_{\mathrm{D}}^{22}=-51.1$ (c 1.00, $\mathrm{CH}_{2} \mathrm{Cl}_{2}$ ), 96\% ee; IR (UATR): 3396, $1693 \mathrm{~cm}^{-1} ;{ }^{1} \mathrm{H}$ NMR (300 MHz, $\left.\mathrm{CDCl}_{3}\right): \delta 6.13(\mathrm{~d}, J=3.1 \mathrm{~Hz}, 1 \mathrm{H}), 5.91(\mathrm{~d}, J=3.1 \mathrm{~Hz}, 1 \mathrm{H})$, $5.16(\mathrm{bs}, 1 \mathrm{H}), 4.82(\mathrm{bs}, 1 \mathrm{H}), 3.90(\mathrm{dd}, J=11.2 \mathrm{~Hz}, J=5.4 \mathrm{~Hz}, 1 \mathrm{H}), 3.83(\mathrm{dd}, J=11.2 \mathrm{~Hz}, J=$ $4.7 \mathrm{~Hz}, 1 \mathrm{H}), 2.61$ (q, $J=7.5 \mathrm{~Hz}, 2 \mathrm{H}), 1.45(\mathrm{~s}, 9 \mathrm{H}), 1.21(\mathrm{t}, J=7.5 \mathrm{~Hz}, 3 \mathrm{H}) ;{ }^{13} \mathrm{C}$ NMR $(75$ $\left.\mathrm{MHz}, \mathrm{CDCl}_{3}\right): \delta 157.8,156.0,150.3,107.7,104.7,80.2,64.9,51.1,28.5,21.5,12.1$; HRMS (ESI, m/z): Calcd. for $\mathrm{C}_{13} \mathrm{H}_{21} \mathrm{NO}_{4} \mathrm{Na}$ : 278.1363, found [M+Na] ${ }^{+}$: 278.1363; HPLC analysis (Chiralpak IA column, heptane $/ 2$-propanol $=97 / 3$, flow rate $=1 \mathrm{~mL} / \mathrm{min}$, wavelength $=220$ $\mathrm{nm}$ ): Rt $=21.60$ (minor) and 23.26 (major).

\subsection{2 (S)-(1-(5-ethyl-4-(4-fluorophenyl)furan-2-yl)-2-hydroxyethyl)carbamic acid tert- butyl ester 14}

$66 \mathrm{mg}(94 \%)$, colorless oil; $[\alpha]_{\mathrm{D}}^{22}=-49.4\left(c 0.50, \mathrm{CH}_{2} \mathrm{Cl}_{2}\right), 97 \% e e$; IR (UATR): 3410 , $1689 \mathrm{~cm}^{-1} ;{ }^{1} \mathrm{H}$ NMR (300 MHz, $\left.\mathrm{CDCl}_{3}\right): \delta 7.32-7.25(\mathrm{~m}, 2 \mathrm{H}), 7.11-7.03(\mathrm{~m}, 2 \mathrm{H}), 6.32(\mathrm{~s}$, 1H), 5.18 (bs, 1H), 4.88 (bs, 1H), 4.00-3.85 (m, 2H), 2.74 (q, J = 7.5 Hz, 2H), 2.14 (bs, 1H), $1.47(\mathrm{~s}, 9 \mathrm{H}), 1.25(\mathrm{t}, J=7.5 \mathrm{~Hz}, 3 \mathrm{H}) ;{ }^{13} \mathrm{C} \mathrm{NMR}\left(75 \mathrm{MHz}, \mathrm{CDCl}_{3}\right): \delta 161.8\left(J_{\mathrm{CF}}^{1}=245.5 \mathrm{~Hz}\right)$, 155.9, 152.5, 150.2, 130.0, $\left(J_{\mathrm{CF}}^{4}=3.3 \mathrm{~Hz}\right), 129.3\left(J_{\mathrm{CF}}^{3}=7.8 \mathrm{~Hz}\right), 120.3,115.6\left(J_{\mathrm{CF}}^{2}=21.4\right.$ $\mathrm{Hz}), 108.7,80.3,64.8,51.1,28.5,20.4,13.0 ;$ HRMS (ESI, m/z): Calcd. for $\mathrm{C}_{19} \mathrm{H}_{24} \mathrm{NO}_{4} \mathrm{FNa}$ : 372.1587, found $[\mathrm{M}+\mathrm{Na}]^{+}:$372.1585; HPLC analysis (Chiralpak IA column, heptane/2propanol $=95 / 5$, flow rate $=1 \mathrm{~mL} / \mathrm{min}$, wavelength $=220 \mathrm{~nm}$ ): $\mathrm{Rt}=15.26$ (minor) and 17.42 (major).

\subsection{2 (S)-2-Amino-2-(5-ethylfuran-2-yl)ethan-1-ol, hydrochloride 15}

To the product $13(47 \mathrm{mg}, 0.18 \mathrm{mmol})$ was added $4 \mathrm{M} \mathrm{HCl}$ in dioxane $(0.5 \mathrm{~mL})$ and let stir for $1 \mathrm{~h}$. The reaction mixture was concentrated in vacuo, taken up with a mixture of diethylether / ethyl acetate (4 / 1). The solid part was separated by filtration, washed with diethylether and dried in vacuum dessicator. Yield: $25 \mathrm{mg}(72 \%)$, beige solid, $\mathrm{mp}=101$ $102^{\circ} \mathrm{C} ;[\alpha]_{\mathrm{D}}{ }^{22}=-16.2$ (c 0.50, MeOH); IR (UATR): 3550-2400 (broad), $2034 \mathrm{~cm}^{-1} ;{ }^{1} \mathrm{H}$ NMR (300 MHz, CD $\left.{ }_{3} \mathrm{OD}\right): \delta 6.43(\mathrm{~d}, J=3.2 \mathrm{~Hz}, 1 \mathrm{H}), 6.07(\mathrm{~d}, J=3.2 \mathrm{~Hz}, 1 \mathrm{H}), 4.41$ (ABX System, $\left.J_{\mathrm{AX}}=8.2 \mathrm{~Hz}, J_{\mathrm{BX}}=4.9 \mathrm{~Hz}, 1 \mathrm{H}\right), 3.94\left(\mathrm{ABX}\right.$ System, $\left.J_{\mathrm{AB}}=11.6 \mathrm{~Hz}, J_{\mathrm{BX}}=4.9 \mathrm{~Hz}, 1 \mathrm{H}\right), 3.88$ $\left(\mathrm{ABX}\right.$ System, $\left.J_{\mathrm{AB}}=11.6 \mathrm{~Hz}, J_{\mathrm{AX}}=8.3 \mathrm{~Hz}, 1 \mathrm{H}\right), 2.66(\mathrm{q}, J=7.5 \mathrm{~Hz}, 2 \mathrm{H}), 1.23(\mathrm{t}, J=7.5 \mathrm{~Hz}$, 
$3 \mathrm{H}) ;{ }^{13} \mathrm{C}$ NMR (75 MHz, $\left.\mathrm{CD}_{3} \mathrm{OD}\right): \delta$ 160.4, 146.9, 111.4, 106.3, 61.9, 51.9, 22.1, 12.5;

HRMS (ESI, m/z): Calcd. for $\mathrm{C}_{8} \mathrm{H}_{11} \mathrm{O}_{2}$ : 139.0759, found [M-NH3+H] ${ }^{+}: 139.0760$.

\section{Acknowledgments}

This research was financially supported by the French Ministry of Higher Education and Research (MESR) and CNRS.

\section{References}

1. (a) Natural products chemistry; Nakanishi, K., Ed.; Kodansha scientific books; Kodansha; Academic Press: Tokyo: New York, 1974; (b) Look, S. A.; Burch, M. T.; Fenical, W.; Zheng, Q.; Clardy, J. J. Org. Chem. 1985, 50, 5741-5746.

2. Lipshutz, B. H. Chem. Rev. 1986, 86, 795-819.

3. (a) Hou, X. L.; Cheung, H. Y.; Hon, T. Y.; Kwan, P. L.; Lo, T. H.; Tong, S. Y.; Wong, H. N. C. Tetrahedron 1998, 54, 1955-2020; (b) Kirsch, S. F. Org. Biomol. Chem. 2006, 4, 2076-2080.

4. (a) Gulevich, A. V.; Dudnik, A. S.; Chernyak, N.; Gevorgyan, V. Chem. Rev. 2013, 113, 3084; (b) Patil, N. T.; Yamamoto, Y. Chem. Rev. 2008, 108, 3395-3442; (c) Hoffmann, M.; Miaskiewicz, S.; Weibel, J.-M.; Pale, P.; Blanc, A. Beilstein J. Org. Chem. 2013, 9, 1774-1780; (d) Dudnick, A. S.; Xia, Y.; Li, Y.; Gevorgyan, V. J. Am. Chem. Soc. 2010, 132, 7645-7655; (e) Wang, E.; Fu, X.; Xie, X.; Chen, J.; Gao, H.; Liu, Y. Tetrahedron Lett. 2011, 52, 1968-1972; (f) Aponick, A.; Li, C.-Y.; Malinge, J.; Marques, E. F. Org. Lett. 2009, 11, 4624-4627; (g) Du, X.; Song, F.; Lu, Y.; Chen, H.; Liu, Y. Tetrahedron 2009, 65, 1839-1845; (h) Blanc, A. ; Tenbrink, K. ; Weibel, J.-M. ; Pale, P. J. Org. Chem. 2009, 74, 5342-5348; (i) Kotikalapudi, R.; Swamy, K. C. K. Tetrahedron Lett. 2012, 53, 3831-3834; (j) Wang, T.; Shi, S.; Rudolph, M.; Hashmi, A. S. K. Adv. Synth. Catal. 2014, 356, 2337-2342; (k) Wang, T.; Shi, S.; Hansmann, M. A.; Rettenmeier, E.; Rudolph, M.; Hashmi, A. S. K. Angew. Chem. Int. Ed. 2014, 53, 3715-3719; (1) Hashmi, A. S. K.; 
Schwarz, L. Chem. Ber./Recueil 1997, 130, 1449-1456; (m) Hashmi, A. S. K.; Ruppert, T. L.; Knöfel, T.; Bats, J. W. J. Org. Chem. 1997, 62, 7295-7304.

5. Paolucci, C.; Rosini, G. Tetrahedron : Asym 2008, 18, 2923-2946.

6. (a) Zhou, W.-S.; Lu, Z.-H.; Xu, Y.-M.; Liao, L.-X.; Wang, Z.-M. Tetrahedron 1999, 55, 11959-11983; (b) Ciufolini, M.; Hermann, C. Y. W.; Dong, Q. ; Shimizu, T.; Swaminathan, S.; Yi, N. Synlett 1998, 105-114; (c) Chen, W. P. ; Roberts, S. M. J. Chem. Soc. Perkin Trans. 1 1999, 103-105.

7. (a) Yi, N. ; Ciufolini, M. A. Tetrahedron Lett. 1995, 36, 6595-6598; (b) Yana, C. F.; Xu, Y. M.; Liao, L. X.; Zhou, W. S. Tetrahedron Lett. 1998, 39, 9227-9228; (c) Achmatowicz, O. ; Bukowski, P.; Szechnev, B. ; Zwierzchowska, Z. ; Zamojski, A. Tetrahedron 1971, 27, 1973-1996.

8. (a) Shono, T.; Matsumura, Y.; Tsubata, J. Chem. Lett. 1981, 1121-1124; (b) Ben-Ishai, D.; Satati, I.; Bernstein, Z. Tetrahedron Lett. 1976, 32, 1571-1573; (c) Kasai, M.; Ziffer, H. J. Org. Chem. 1983, 48, 2346-2349.

9. (a) Demir, A. S.; Sesenoglu, O.; Uelkue, D.; Arıcı, C. Helv. Chim. Acta 2003, 86, 91-105; (b) Bushey, M. L.; Hankaas, M. H.; O’Doherty, G. A. J. Org. Chem. 1999, 64, 29842985; (c) Alvaro, G. ; Martelli, G. ; Savoia, D. ; Zuffoli, A. Synthesis 1998, 1773-1777; (d) Irako, N.; Hamada, Y. ; Shioiri, T. Tetrahedron 1995, 51, 12731-12744; (e) Zhou, W. S.; Lu, Z. H.; Wang, Z. M. Tetrahedron Lett. 1991, 32, 1467-1470. For the synthesis of the next higher homologous by enzyme catalysis see: Hashmi, A. S. K.; Ata, F.; Haufe, P.; Rominger, F. Tetrahedron 2009, 65, 1919-1927.

10. (a) Gouault, N.; Le Roch, M.; Cornée, C. ; David, M. ; Uriac, P. J. Org. Chem. 2009, 74, 5614-5617; (b) Gouault, N.; Le Roch, M. ; Cheignon, A. ; Uriac, P.; David, M. Org. Lett. 2011, 13, 4371-4373; (c) Trinh, T. T. H. ; Nguyen, K. H. ; de Aguiar Amaral, P. ; Gouault, N. Beilstein J. Org. Chem. 2013, 9, 2042-2047. 
11. Campbell, A.; Raynham, T.; Taylor, K. Synthesis 1998, 12, 1707-1709.

12. (a) Alfaro, R.; Yuste, F.; Ortiz, B.; Sanchez-Obregon, R.; Garcia Ruano, J. L. Tetrahedron 2009, 65, 357-363; (b) Xu, G. G.; Etzkorn, F. A. Org. Lett. 2010, 12, 696699; (c) Song, X.-N.; Yao, Z.-J. Tetrahedron 2010, 66, 2589-2593.

13. Takai, K.; Nitta, K.; Utimoto, K. D. J. Am. Chem. Soc. 1986, 108, 7408-7410.

14. (a) Fu, Y.; Bieschke, J.; Kelly, J. W. J. Am. Chem. Soc. 2005, 127, 15366-15367; (b) Kaltenbronn, J. Hudspeth, J. P.; Lunney, E. A.; Michniewicz, B. M.; Nicolaides, E. D.; Repine, J. T.; Roark, W. H.; Stier, M. A.; Tinney, F. J.; Woo, P. K. W.; Essenburg, A. D. J. Med. Chem. 1990, 33, 838-845.

15. D’Aniello, F.; Mann, A.; Schoenfelder, A.; Taddei, M. Tetrahedron 1997, 53, 1447-1456.

16. (a) El Sohly, A. M.; Wespe, D. A.; Poore, T. J.; Snyder, S. A. Angew. Chem., Int. Ed. 2013, 52, 5789-5794; (b) Pawluc, P.; Franczyk, A.; Walkowiak, J.; Hreczycho, G.; Kubicki, M.; Marciniec, B. Tetrahedron 2012, 68, 3545-3551.

17. (a) Reddy, J. S.; Rao, B. V. J. Org. Chem. 2007, 72, 2224-2227; (b) Aoki, S.; Matsui, K.; Tanaka, K.; Satari, R.; Kobayashi, M. Tetrahedron 2000, 56, 9945-9948.

18. Walsh, P. J.; Sharpless, K. B. Synlett 1993, 605-606.

19. Nguyen, K. H.; Tomasi, S.; Le Roch, M.; Toupet, L.; Renault, J.; Uriac, P.; Gouault, N. J. Org. Chem. 2013, 78, 7809-7815.

20. (a) Miyaura, N.; Suzuki, A. Chem. Rev. 1995, 95, 2457-2483; (b) Suzuki, A. J. Organomet. Chem. 1999, 576, 147-168.

21. Heck, R. F. J. Am. Chem. Soc. 1968, 90, 5518-5526.

22. Sonogashira, K.; Tohda, Y.; Hagihara, N. Tetrahedron Lett. 1975, 16, 4467-4470.

23. (a) Guram, A. S.; Rennels, R. A.; Buchwald, S. L. Angew. Chem., Int. Ed. 1995, 34, 13481350; (b) Louie, J.; Hartwig, J. F. Tetrahedron Lett. 1995, 36, 3609-3612. 